\title{
Toward Smart Building Design Automation: Extensible CAD Framework for Indoor Localization Systems Deployment
}

\author{
Andrea Cirigliano, Roberto Cordone, Alessandro A. Nacci, and \\ Marco Domenico Santambrogio, Senior Member, IEEE
}

\begin{abstract}
Over the last years, many smart buildings 2 applications, such as indoor localization or safety systems, have 3 been subject of intense research. Smart environments usually rely 4 on several hardware nodes equipped with sensors, actuators, and 5 communication functionalities. The high level of heterogeneity 6 and the lack of standardization across technologies make design 7 of such environments a very challenging task, as each instal\& lation has to be designed manually and performed ad-hoc for 9 the specific building. On the other hand, many different systems 10 show common characteristics, like the strict dependency with 11 the building floor plan, also sharing similar requirements such 12 as a nodes allocation that provides sensing coverage and nodes 13 connectivity. This paper provides a computer-aided design appli14 cation for the design of smart building systems based on the 15 installation of hardware nodes across the indoor space. The 16 tool provides a site-specific algorithm for cost-effective deploy17 ment of wireless localization systems, with the aim to maximize 18 the localization accuracy. Experimental results from real-world 19 environment show that the proposed site-specific model can 20 improve the positioning accuracy of general models from the ${ }_{21}$ state-of-the-art. The tool, available open-source, is modular and 22 extensible through plug-ins allowing to model building systems 23 with different requirements.
\end{abstract}

24 Index Terms-Indoor localization, Internet of Things, 25 performance optimization, smart buildings design automation.

26

$\mathbf{O}$ N AVERAGE, people spend approximately $70 \%$ of their time indoors [1], such as in offices, schools, and at 29 home. New indoor smart applications are being developed 3o at high rate, in both research and commercial areas cover${ }_{31}$ ing a wide range of personal and social scenarios. Smart 32 buildings are becoming a reality with the adoption of an under${ }_{33}$ lying monitoring and communication infrastructure composed 34 by access points (APs), sensor motes, cameras, and smart 35 devices integrated in a building management systems (BMSs).

Manuscript received September 14, 2016; accepted November 13, 2016. This paper was recommended by Associate Editor S. Mohanty.

A. Cirigliano, A. A. Nacci, and M. D. Santambrogio are with the Department of Electronics, Information and Bioengineering, Politecnico di Milano, 20133 Milan, Italy (e-mail: andrea.cirigliano@mail.polimi.it).

R. Cordone is with the Department of Computer Science, Universita' degli Studi di Milano, 20100 Milan, Italy.

Color versions of one or more of the figures in this paper are available online at http://ieeexplore.ieee.org.

Digital Object Identifier 10.1109/TCAD.2016.2638448
The BMS is a control system that monitors the building state 36 and operates through actuators to increase the comfort and ${ }_{37}$ safety of occupants, while managing the energy efficiency at 38 the same time.

Many smart buildings applications are based on indoor 40 localization techniques, using location information to optimize ${ }_{41}$ the environment and provide context-aware services. Indoor 42 localization systems often require the presence of wireless 43 devices such as APs, in order to let the user identify its position ${ }_{44}^{4}$ by means of a mobile device. Most smart building applica- 45 tions have been developed in order to achieve sustainability, reducing energy waste related to energy-consuming appliances like heating, ventilation, and air-conditioning (HVAC). Some examples are [2] and [3]. Smart HVAC systems usually rely on a set of ambient sensors able to collect indoor values of 50 temperature and humidity. This allows the control system to 51 build thermal maps of the indoor environment, locate thermal 52 complaint feedbacks coming from the tenants and regulate ${ }_{53}$ only the necessary portion of the physical system. Another 54 target feature of complex buildings is safety, characterized by 55 the ability to respond to crisis events limiting damages and 56 victims. These systems are able to detect safety threats, for ${ }_{57}$ example from smoke detectors or heat detectors. Also in this scenario, a proper allocation of sensor nodes is essential to detect and locate the threat responsively.

The position of each node strongly affects the performance 61 of the system, since a bad allocation could lead to unmonitored 62 areas. The number of nodes employed, besides weighting on 63 the installation cost, also burdens the overall energy consump- 64 tion of the system, a key parameter to consider especially for 65 energy saving systems. The choice of the hardware nodes can 66 get more difficult by the availability on the market of several ${ }_{67}$ devices and components that differ in cost, power consumption 68 and maximum range distance. Although the key role of nodes allocation, many smart building systems proposed in literature do not consider nodes amount and positioning problems in environments that differ from the original testbeds.

Without a systematic approach the design space is not well 73 explored, which leads to inefficient solutions. In this con- 74 text, the development of tools able to automatize part of the 75 design flow of smart building systems is essential. In order 76 to find a near-optimal allocation of nodes, the knowledge of 77 the floor plan is required. However, for installations performed 78 on existing buildings, administrators can encounter difficulties 79 
TABLE I

\begin{tabular}{|l|c|c|c|c|}
\hline \multicolumn{1}{|c|}{ Deployment } & Site Specific vs General Model & Heterogeneous Nodes & Application Integrated & Extensible \\
\hline Zhao et al. [4] & General Model & No & Yes & No \\
\hline He at al. [5] & General Model & No & No & No \\
\hline Fang et. al. [6] & Site Specific Model & No & No & No \\
\hline Proposed approach & Site Specific Model & Yes & Yes & Yes \\
\hline
\end{tabular}

80 in obtaining the floor plan in an easily-interpretable digital 81 format.

82 To address these problems, we developed a computer-aided 83 design (CAD) tool to assist building designers during the 84 design of smart building systems. The application manages 85 common requirements like the building floor plan specifica86 tion. We decided to implement a node allocation algorithm 87 for three different indoor localization systems, that searches for 88 near-optimal allocations of nodes, from mixed hardware types, 89 with the aim of keeping low the total cost. Due to the high level 90 of heterogeneity and lack of standardization across systems to 91 design, we make the system extensible through plug-ins to let 92 new functionalities being integrated into the system. The tool ${ }^{1}$ ${ }_{93}$ is developed within the $\mathrm{QCAD}^{2}$ environment, an open-source 94 computer-aided drafting application. The key contributions of 95 this paper can be summarized as follows.

\section{RELATED WORK}

Building information modeling (BIM) is a consolidate 111 process to support building constructions and renovations. 112 BIM softwares, and in particular CAD for buildings such as ${ }_{113}$ ArchiCAD [7], focus on the generation and management of 114 digital representations of the physical aspects of places. BIM 115 tools can coordinate architectural and structural requirements, 116 for essential tasks such as collision detection [8]. Materials 117 employed for a construction can be represented with extremely 118 high levels of accuracy, thanks to the several libraries devel119 oped in many years, resulting in precise cost estimations [9]. 120 With the diffusion of integrated smart systems built to increase 121 comfort and efficiency, buildings require the design of aspects 122 that go beyond the mere physical design. The concept of smart

\footnotetext{
${ }^{1} \mathrm{~A}$ video demo of the tool has been published at https://youtu.be/6c6D6wolDBQ.

${ }^{2}$ QCAD—Open Source CAD System: http://www.qcad.org/.

${ }^{3}$ The source code of the system is open-source and available at https://bitbucket.org/necst/box-smartcad.
}

environment is becoming more and more concrete with the ${ }_{123}$ integration of sensors, actuators and computational elements 124 in buildings, while tools able to model smart and interactive ${ }_{125}$ functionalities of modern buildings are currently lacking. $\quad 126$

The problem of the allocation of hardware nodes in a given ${ }^{127}$ environment can be compared, on first approximation, by the ${ }_{128}$ maximal cover location problem (MCLP), i.e., the problem ${ }_{129}$ of covering the maximum amount of demand locations with 130 a given number of facilities. Similarly, the location set cov- ${ }_{131}$ ering problem (LSCP) consists in finding the minimum set ${ }_{132}$ of facilities that covers all available demand locations. Each ${ }_{133}$ facility has the same coverage radius $r$; a demand point is ${ }_{134}$ assumed to be covered if it is within distance $r$ of a facil- ${ }_{135}$ ity. Daskin et al. [10], [11] gave a general formulation of the 136 LSCP and reformulated it for network systems and emergency ${ }_{137}$ vehicle deployment.

The maximum sensing coverage region is a special case ${ }_{139}$ of the previous two problems that focuses on the research of 140 an allocation of wireless nodes that guarantees both sensing 141 coverage and network connectivity between nodes [12], [13]. 142 In this scenario, the placement need to take care not only of 143 the sensing range, but also of the communication range of each 144 node.

For what concern the allocation in indoor environments, 146 only minimum literature has been published so far to the ${ }_{147}$ best of our knowledge. Zhao et al. [4] proposed an AP posi- 148 tioning model based on the differential evolution algorithm, 149 specific for fingerprinting localization techniques. Their model 150 focuses on increasing the diversity of the received signal array 151 along the indoor locations, and thus improving the position- 152 ing accuracy of fingerprinting schemes. However, the model ${ }_{153}$ does not take into account the effect of walls or other obsta- 154 cles present in the target environment. He et al. [5] made use 155 of a genetic algorithm for APs deployment model, to study 156 the relationship between positioning error and signal space ${ }_{157}$ Euclidean distance. Again, the simulation results show that ${ }_{158}$ the error can be reduced increasing the Euclidean distance ${ }_{159}$ between the received signal strength (RSS) arrays of differ- 160 ent locations. Fang and Lin [6] proposed a tool for linking 161 the placement of APs and the positioning performance. Their ${ }_{162}$ algorithm maximizes signal-to-noise ratio, i.e., maximizes the ${ }_{163}$ signal and minimizes the noise simultaneously. However, the ${ }_{164}$ system is developed in a real-world environment, and requires 165 measurements with different AP allocations that can be an 166 expensive and time-consuming task.

A common limitation of many works described previously is 168 the employment of simple and general models which does not ${ }_{169}$ take into account the actual layout and geometry of the build- 170 ing. The free-space path loss propagation model is often used 171 


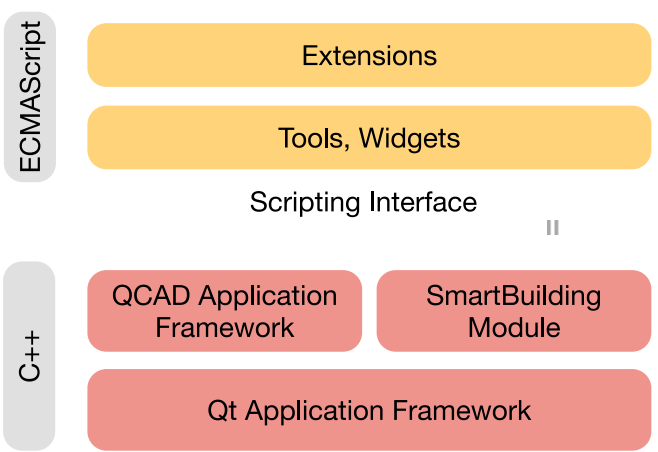

Fig. 1. Overview of the application stack. The script interpreter features standard ECMAScript functionality and on top of that provides additional classes from the Qt API, QCAD API, and the SmartBuilding module.

172 despite the presence of fixed obstructing objects like walls. Of 173 course, none of the cited works provide a convenient way to 174 specify geometric layout of the indoor environment. This leads 175 the authors to validate models simply using squared or rectan176 gular areas to represent the indoor environment, omitting the 177 relationship between irregular areas and system coverage. In 178 addition, none of the existing solutions takes in consideration 179 different hardware characteristics and costs of the nodes to be 180 deployed.

\section{Proposed Application Framework}

182 Our system has been developed on top of the QCAD appli183 cation framework. The QCAD application framework consists 184 of programming libraries and resources that provides CAD 185 specific functionalities. An example of module provided by 186 the QCAD application framework is the Math module that 187 implements mathematical concepts such as vectors or matri${ }_{188}$ ces as well as basic geometrical classes like points, lines and 189 so on. The QCAD Framework has been enhanced with a 190 SmartBuilding module that provides some fundamental func191 tionalities for the design of smart building systems. The 192 module include abstract entities like rooms, walls, sockets, 193 sensor nodes and gateways. User interface components are also 194 provided in order to create and edit this entities (tools) and to 195 specify parameters (widgets). Our module implements a node 196 deployment algorithm for three commons indoor localization 197 systems, that will be discussed later. The whole application 198 rely on Qt, a framework that covers a lot of generic and low199 level functionality for desktop applications and not directly 200 related to CAD.

201 The QCAD application framework offers a very complete 202 and powerful ECMAScript interface. The SmartBuilding mod203 ule, as well as the QCAD application framework, is accessible 204 through that scripting interface. Through the ECMAScript 205 interface developers will be able to extend the whole appli206 cation in an easy and very efficient way. The choice of a 207 popular script language that is easy to learn enables anyone 208 with previous programming experience to extend the appli209 cation. Such extensions can for example be CAD related 210 interactive tools like an HVAC layout construction widget, or 211 a temperature sensor nodes deployment algorithm.

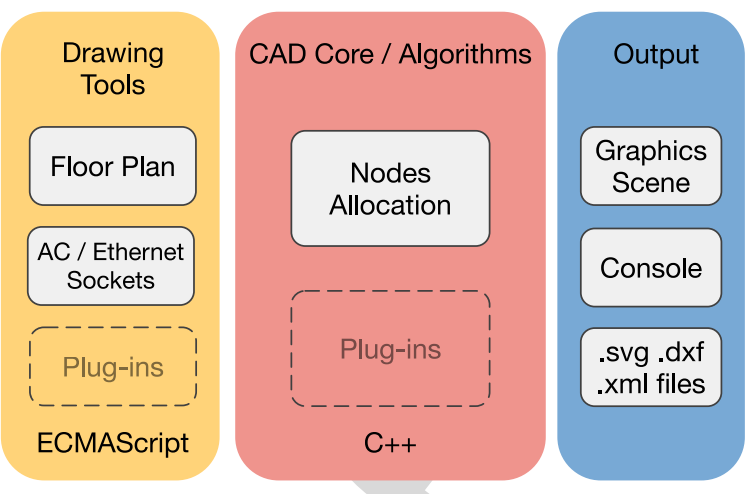

Fig. 2. Functional overview of the system components. Drawing tools and algorithms for systems deployment and simulation are extensible through ECMAScript or $\mathrm{C}++$ plug-ins.

In some situations extending QCAD through scripts alone 212 may not be possible. This is mostly the case, if the extension ${ }^{213}$ is based on an existing $\mathrm{C}$ or $\mathrm{C}++$ library. In that case, it is 214 possible to create a $\mathrm{C}++$ plug-in that wraps the existing library 215 and adds the necessary hooks to access library functionality 216 through the script interface. Such a plug-in will be automati- ${ }^{217}$ cally loaded by QCAD on start up to add functions and classes ${ }_{218}$ to the script interface of QCAD. These script extensions can ${ }_{219}$ then be used by a script add-ons to make that functionality 220 available as part of the application interface.

\section{NODES DePloyment FOR INDOOR LOCALIZATION}

Smart environments always rely on a set of hardware nodes 224 able to collect sensing data and communicate through cabled 225 or wireless technologies. The number of nodes employed and 226 the position of each one strongly affect the overall performance 227 of the system as well as the cost of installation. In this paper, 228 indoor localization systems have been taken as the main case 229 study for the nodes allocation, since occupants localization 230 and monitoring is one of the most common requirements of 231 different smart environments.

232

The way in which the indoor environment must be cov- 233 ered by the nodes depends on the particular technology 234 implemented; however, there can be identified three main ${ }_{235}$ manners.

1) Single coverage, i.e., to monitor the state of the envi- 237 ronment with a single node for each location inside its 238 radius. This includes for example to detect the presence 239 of a mobile device in a proximity region [14], or to 240 detect an RFID tag within the tags reader range [15]. ${ }_{241}$

2) Trilateration, to compute the position of a mobile device. 242 This technique requires the reception of a wireless signal 243 of at least three reference sensors with well-known posi- 244 tions everywhere within the covered area. We define the 245 term $k$-coverage as the minimum number of sensors (or 246 reference nodes) required in each location by a system. ${ }_{247}$ Single coverage systems have $k$-coverage $=1$, while for 248 trilateration $k=3$.

3) Fingerprinting, where the number and the strength of the 250 received signals is not fixed, but affect the localization 251 accuracy. 


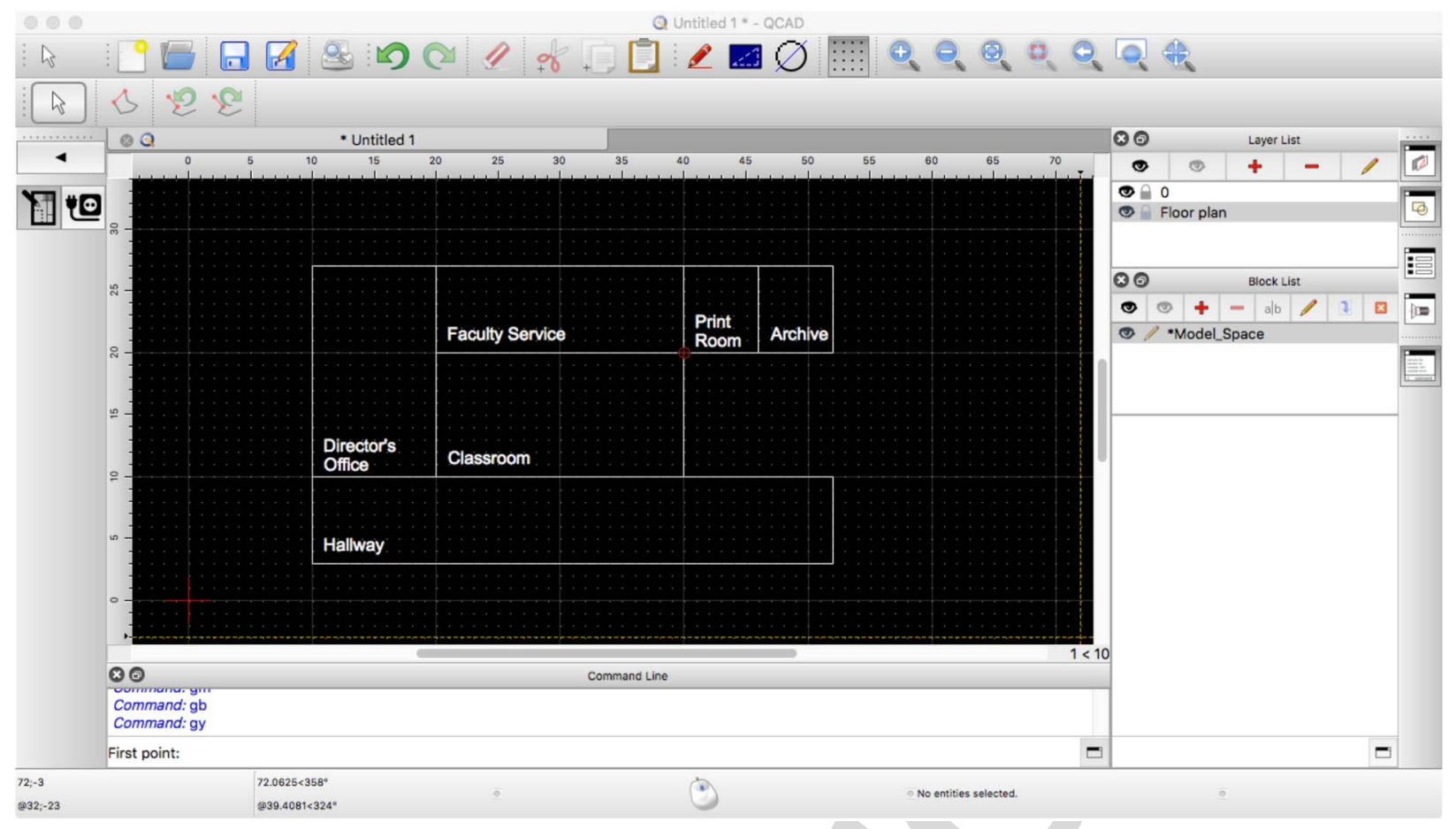

Fig. 3. Floor-plan design tool. User can specify the layout of the rooms and a possible set of candidate sites for the node placement.

253 Trilateration and fingerprinting usually exploit wireless tech254 nologies as Wi-Fi or Bluetooth to establish a connection 255 between mobile and stationary nodes. Sensing regions can 256 refer to any type of ambient sensors, such as passive infrared 257 sensors [16], remote thermal sensors [17], but also proximity258 based radio transmitters such as RFID tag readers [18] and 259 Bluetooth low energy transmitters (BLE beacons) [19].

\section{0}

\section{Proposed Deployment ToOl}

261 As we previously said, smart environments always rely on 262 a set of sensor nodes, each one able to communicate through 263 cabled or wireless technologies. Also for outdoor WSNs, a key 264 challenge is how to achieve coverage of the target monitor265 ing space and sufficient network connectivity between sensor 266 nodes. Usually each sensor mote communicates with the rest 267 of the network through technologies like Wi-Fi or ZigBee. 268 Additional issues for outdoor WSNs are the limited battery life 269 of each node and the power consumption required for packet 270 transmissions. Given the availability in most (also "nonsmart") 271 buildings of power outlets, Ethernet sockets and Wi-Fi sig272 nal, the mentioned limitations of WSNs can be solved in 273 indoor application making use of the existing infrastructure. 274 Differently from outdoor WSN deployments, where cover275 age and connectivity are always treated together, our system 276 leaves nodes connectivity optional, focusing on providing the 277 coverage service to the indoor locations.

278 The design process starts with a drafting phase in which the 279 user specify the building floor plan as a set of rooms. During 280 this phase the designer can restrict the possible sites for nodes 281 allocation, selecting a set of candidate points. This can be 282 useful when the hardware devices require power supply or
Ethernet connectivity. The design interface used for both map 283 and candidate sites specification is reported in Fig. 3.

In our model, we will refer to $L$ as the entire set of monitor- 285 ing locations to be covered, while $J$ as the set of deployable 286 locations where nodes can be placed. By default, $L=J$ and ${ }_{287}$ nodes can be positioned everywhere but as we said the set $J{ }_{288}$ can be restricted only to specific candidate points. 289

After the design phase, different parameters are provided by 290 the administrator and used to define a domain in which search 291 for a covering solution. The parameters are as follows. 292

1) The covering technique (single, trilateration, or fin- 293 gerprinting) that will be used to cover the locations 294 in $L$.

2) A cost $c_{t}$ for every type $t \in T$ of node available on the 296 market (expressed in dollars).

3) A working range $r_{t}$ for every type $t$ of node (expressed 298 in meters).

4) A percentage of covered area required, called target (i.e., 30 the minimum percentage of locations $l \in L$ to be covered 301 by the solution).

The system will return to the designer a set $N$ of nodes $n_{j t}{ }_{303}$ (possibly with mixed hardware types) and their position on 304 the building map. The outcome will have the lower cost of 305 installation among all the inspected solutions that satisfy the 306 target percentage of covered area. Fig. 4 shows an overview 307 of the process explained so far.

\section{A. Covering Techniques}

Our tool provides three different ways to cover the floor- 310 plan space, each one identified by the technique required by 311 the system that will be installed. 


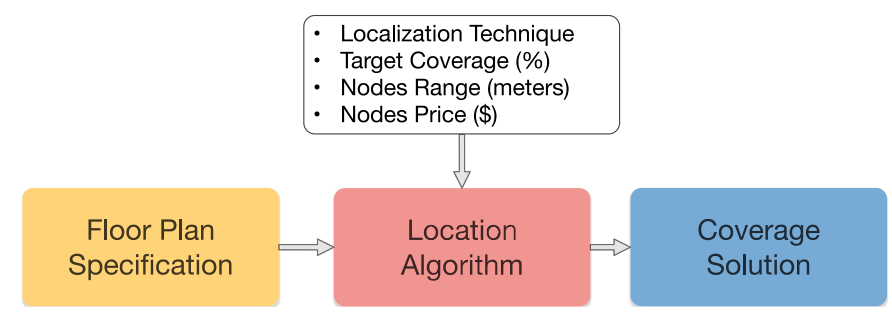

Fig. 4. System process. After the design of the floor plan, different parameters are used to define the search for an optimal allocation of nodes.

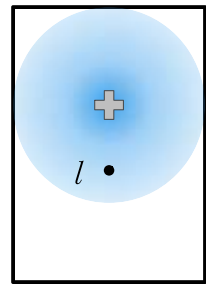

(a)

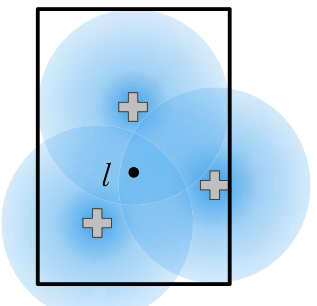

(b)

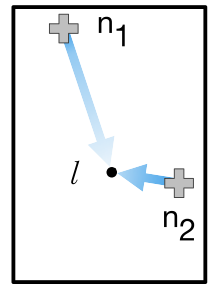

(c)

Fig. 5. Sample floor-plans with a location $l$ covered (a) in single mode, (b) for trilateration, and (c) for fingerprinting where $\operatorname{rss}_{l, 1}<\operatorname{rss}_{l, 2}$.

1) Single coverage that guarantees from each position the presence of at least one reachable node. This is used for example to detect the presence of a mobile device in a proximity region. In our model, a location $l$ of the floorplan is considered covered if exists at least one working node $n$ of type $t$ within a range $r_{t}$. An example is shown in Fig. 5(a).

2) Trilateration: This is the process of determining the position of a point measuring its distance from three reference nodes, exploiting geometric properties of triangles. Usually, indoor trilateration systems use the strength of the signal received from a node to estimate its distance. In our model, a location $l$ of the floor-plan is covered for trilateration if there exist at least three working nodes $n_{1}, n_{2}$, and $n_{3}$, each one no more distant then its corresponding range $r_{t}$. A location $l$ served for trilateration is shown in Fig. 5(b). Although we refer only to trilateration, the same exact result can be used also for triangulation, the technique where angles are measured instead of distances.

3) Fingerprinting: This technique is used to estimate the position of a mobile device based on its rss vector. Each location receives the signal from $k$ nodes, where $k$ is not the same for all locations, but depends on how many nodes are reachable from that particular location. Each one of the $k$ signals reaches the receiving antenna with a given power (or rss). For example, the location $l$ shown in Fig. 5(c) perceives $k=2$ signals so that $\operatorname{rss}_{l, 1}<\operatorname{rss}_{l, 2}$. We denote as $\operatorname{rss}_{l, n}$ the signal strength received at location $l$ from a node $n$. The vector $\operatorname{rss}_{\mathbf{I}}=\left[\operatorname{rss}_{l, 1}, \ldots, \operatorname{rss}_{l, k}\right]$ of the $k$ signals received at run-time in location $l$ is compared with a dataset of vectors, each one prelabeled with the corresponding position.
The comparison is usually performed by a classification 346 algorithm using the Euclidean distance of the vectors, since ${ }_{347}$ rss vectors with a small Euclidean distance between them are 348 more likely to be close also in the physical space. We have ${ }_{349}$ defined as $\operatorname{rss}_{l, n}$ the signal strength received at location $l$ from 350 a node $n$. The Euclidean distance between $\operatorname{rss}_{\mathbf{a}}$ and $\operatorname{rss}_{\mathbf{b}}$, both 351 composed by $k$ received signals, and collected, respectively, 352 in location $a$ and $b$ is defined as

$E(a, b)=\sqrt{\left(\operatorname{rss}_{a, 1}-\operatorname{rss}_{b, 1}\right)^{2}+\cdots+\left(\operatorname{rss}_{a, k}-\operatorname{rss}_{b, k}\right)^{2}}$.

Consider the vector $\mathrm{rss}_{\mathbf{a}}$ as the run-time sample, while 355 the vector $\mathrm{rss}_{\mathbf{b}}$ retrieved from the stored fingerprint. The 356 smaller is the $E(a, b)$, more confident is the localization system ${ }_{357}$ approximating current location of $a$ with the stored location ${ }_{358}$ of $b$.

It has been demonstrated that maximizing the Euclidean 360 distances of the rss arrays between all sampling points, the 361 positioning accuracy of wireless localization systems can be 362 improved [4], [5]. Fig. 6 is reported a graphical demonstra- 363 tion of the aforementioned statement. Take as an example a 364 dataset (DS1, DS2, DS3, DS4) of stored rss vectors, where ${ }_{365}$ each vector is bi-dimensional $(K=2)$ and coupled with the 366 corresponding physical position. Fig. 6(a) shows each element ${ }_{367}$ of the database where the Cartesian coordinates corresponds to ${ }_{368}$ components $\mathrm{rss}_{1}, \mathrm{rss}_{2}$. Although the plane does not represent ${ }_{369}$ the physical area of the floor-plan, database elements that are 370 near between them are more likely to be close also in the phys- 371 ical space. Given a run-time element $R$, each arrow represents 372 the Euclidean distance $E\left(R, \mathrm{DS}_{i}\right)$ from the surrounding dataset ${ }_{373}$ elements. A localization algorithm can exploit the Nearest ${ }_{374}$ Neighbor technique to approximate the position of $R$ with 375 the nearest dataset element. Unfortunately, the run-time rss 376 measurement of $R$ will not be constant over time, but will 377 experience continuous fluctuations due to environmental noise. 378 These fluctuations make the sample $R$ move randomly to the 379 surrounding points. Suppose that DS2 is the nearest points to 380 $R$ in the physical space. Fig. 6(b) shows with a green area the ${ }_{381}$ probability to assign $R$ the correct (or more accurate) position, 382 while a red (with line pattern) area represents the probability ${ }_{383}$ to get a wrong position from the system. Fig. 6(c) demon- 384 strates how an increase in the rss Euclidean distance between 385 sampling points increase the red area and the accuracy of the 386 localization, while in Fig. 6(d) an Euclidean distance reduction ${ }_{387}$ will lead to poorer localizations.

The RSS has been estimated using the The WINNER II 389 path loss model [20]

$$
P L=A \log _{10}(d[m])+B+C \log _{10}\left(\frac{f_{c}[\mathrm{GHz}]}{5.0}\right)+X
$$

where $P L$ is the signal path loss (in $\mathrm{dB}$ ), $f_{C}$ is the frequency 392 in $\mathrm{GHz}$, and $d$ is the distance between the transmitter and ${ }_{393}$ the receiver location in meters. Values of coefficients $A, B, C, 394$ and $X$ change depending on line-of-sight (LOS) or nonline-of- 395 sight (NLOS) propagations, and are reported in Table II. The 396 propagation model has been used in fingerprinting coverage to 397 maximize the Euclidean distance of the rss vectors between a 398 location and its surrounding points, with the aim of improve 399 the localization accuracy of the system. 


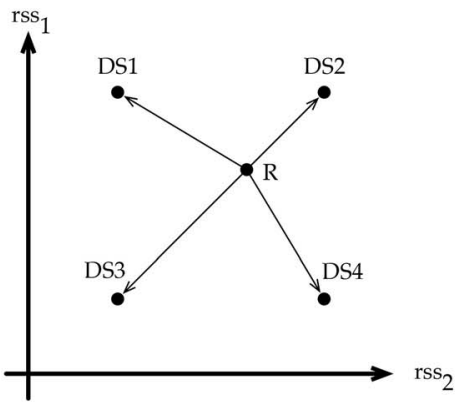

(a)

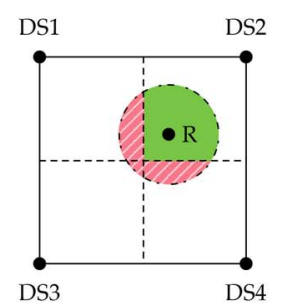

(b)

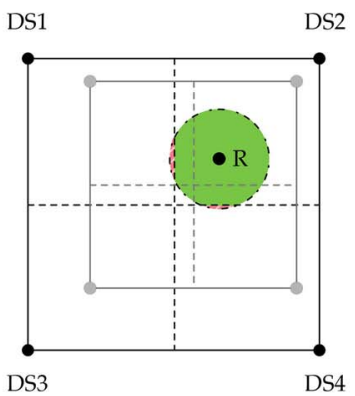

(c)

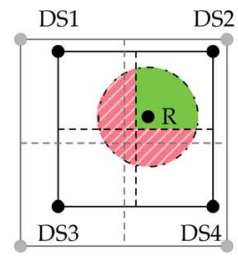

(d)

Fig. 6. (a) Bi-dimensional elements of the localization dataset are represented in Cartesian coordinates corresponding to components rss 1 and rss2. A run-time sample $R$ is shown in (b) where its circular area delineates run-time signal fluctuations. If DS2 is the nearest points to $R$ in the physical space, green area is proportional to the probability of correct localization, while red dashed area represent wrong localizations. (c) Euclidean distance between sampling points has been increased, improving the correct localization. (d) Opposite effect.

TABLE II

VALUES OF COEFFICIENTS DEPENDING ON LOS OR NLOS Propagations. Values have Been Taken From THE WINNER II PATH LOSS MODEL [20]

\begin{tabular}{|l|l|}
\hline Scenario & \multicolumn{1}{|c|}{ Path Loss Coefficients } \\
\hline LOS & $A=18.7, B=46.8, C=20$ \\
\hline \multirow{3}{*}{ L OS } & $A=36.8, B=43.8, C=20$ \\
& $X=5\left(n_{w}-1\right)$ (light walls) \\
& $X=12\left(n_{w}-1\right)$ (heavy walls) \\
\hline
\end{tabular}

401 The 2-D space of the floor plan is discretized with a length 402 unit (default is $1 \mathrm{~m}$ ) that is chosen by the user during the map 403 specification phase.

404 As we have said, in addition to location coverage, also nodes 405 connectivity has been modeled. In our model, a sensor node $n$ 406 is connected if exist a connected path to the gateway node. To 407 ensure the connectivity of the whole network, the following 408 equation must hold:

$409 \quad \forall n \in N$, connected $(n$, gateway $)=$ true

410 where

$$
\begin{aligned}
& 411 \\
& 412 \\
& 413 \\
& 414 \\
& 415
\end{aligned}
$$$$
416
$$
417 rithm in the same way of nonconnected networks, with the 418 following exception.

419 1) First, a manual gateway nodes allocation is required.

420 2) During nodes allocation, deployable points $421 \quad J$ are restricted to locations $j^{\prime}$ such that $422 \quad \operatorname{connected}\left(n_{j^{\prime}}\right.$, gateway $)=$ true.

423 3) During deployment optimization, nodes moves are considered feasible only within the connected area.

\section{COVERing Location Algorithm}

426 The covering location algorithm has the purpose of plac427 ing an optimal set of nodes on the building floor plan.
TABLE III

Notation and Meaning of Symbols Used for the Model

\begin{tabular}{|l|l|}
\hline Notation & \\
\hline$L$ & set of monitoring locations \\
\hline$J$ & set of deployable locations \\
\hline$c_{t}$ & cost of a node of type $t$ \\
\hline$r_{t}$ & sensing range of a node of type $t$ \\
\hline$h_{t}$ & communication range of a node of type $t$ \\
\hline$t a r g e t$ & coverage rate of $L$ required by user $(\%)$ \\
\hline$n_{j t}$ & nodes of type $t$ allocated in $j$ \\
\hline$r s s_{l, n}$ & signal strength received in $l$ from $n$ \\
\hline rss $\mathbf{a}$ & vector of all the $r s s_{a, n}$ values collected in $a$ \\
\hline$E(a, b)$ & Euclidean distance between rss $\mathbf{a}$ and rss $_{\mathbf{b}}$ \\
\hline$D_{l}$ & set of locations no more distant than $d$ from $l$ \\
\hline$z$ & average signal space Euclidean distance \\
\hline$Z$ & objective function \\
\hline$b_{l}$ & reward earned for covering location $l$ \\
\hline$w_{l}$ & reward weighted on the node cost \\
\hline$x_{j t}$ & allocation of node with type $t$ in $j$ (binary) \\
\hline$a_{l j t}$ & reachability of $n_{j t}$ from location $l$ (binary) \\
\hline$k$-coverage & number of ref. nodes required by the system \\
\hline$k_{l}$ & current number of ref. nodes covering $l$ \\
\hline$S$ & min. signal space Euclidean distance threshold \\
\hline$s_{\min }$ & minimum number of node moves in shaking procedure \\
\hline$s_{\max }$ & maximum number of node moves in shaking procedure \\
\hline$R m a x$ & number of restarts of the $V N$ algorithm \\
\hline
\end{tabular}

We have decided to implement a modified version of the 428 multimode covering location problem [21], a generalization ${ }_{429}$ of the MCLP. Using a quite general and flexible reformu- 430 lation of the covering problem, we have been able to adapt 431 the algorithm at the different covering techniques described ${ }_{432}$ previously.

The positioning algorithm is composed by a first Greedy 434 procedure, whose solution is then improved by a variable 435 neighborhood search (VNS) algorithm. The positioning algo- 436 rithm evaluates different solutions using a reward $b_{l}$, that is 437 defined for each location $l$ and will be earned only for the 438 locations covered in that particular solution. The value of the 439 reward depends on the coverage technique.

1) Single Coverage: The reward $b_{l}$ will be earned if there ${ }_{441}$ is at least one node that covers $l$.

2) Trilateration: The reward $b_{l}$ will be earned if there are 443 at least three nodes that cover $l$. 41 42 . 443 5 (n) . (n) 440 


$$
\begin{gathered}
\mathbf{r s s}_{\mathbf{l}}=\left\langle r s s_{l, 1}, r s s_{l, 2}\right\rangle=\langle-84,-72\rangle[d B] \\
\mathbf{r s s}_{\mathbf{s}}=\left\langle r s s_{s, 1}, r s s_{s, 2}\right\rangle=\langle-67,-41\rangle[d B] \\
E(l, s)= \\
=\sqrt{\left(r s s_{l, 1}-r s s_{s, 1}\right)^{2}+\left(r s s_{l, 2}-r s s_{s, 2}\right)^{2}}= \\
=\sqrt{(-84-(-67))^{2}+(-72-(-41))^{2}}= \\
=35.36[d B]
\end{gathered}
$$

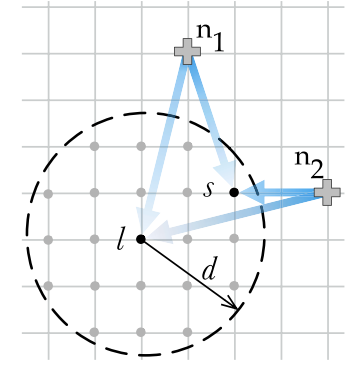

Fig. 7. Regular grid showing how is computed the mean Euclidean distance between the received rss vectors in a certain location $l$, and the surrounding locations $s$ within a certain distance $d$.

451 racy of the system it is possible to increase the signal space ${ }_{452}$ Euclidean distance between the target points. Consider the 453 mean Euclidean distance between the received rss vector in 454 a certain location $l$, and the surrounding locations $s$ within a 455 certain distance $d$

456

457

$$
\begin{aligned}
& \frac{1}{\left|D_{l}\right|} \sum_{s \in D_{l}} E(l, s) \\
& D_{l}=\{s \in L \mid \operatorname{distance}(l, s) \leq d\} .
\end{aligned}
$$

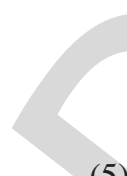

${ }_{458}$ The distance $d$ is used to restrict the rss comparison and 459 diversification only to the locations that are more likely to be ${ }_{460}$ erroneously confuse with $l$ by the localization system. Fig. 7 461 shows an example of how the Euclidean distance of a location 462 is compared to a neighbor location.

${ }_{463}$ We define the average signal space Euclidean distance $z$

$$
z=\frac{\sum_{l \in L} \sum_{s \in D_{l}} \frac{E(l, s)}{\left|D_{l}\right|}}{|L|}
$$

465 The term $z$ will be used by the Greedy procedure to produce 466 a first solution with a reasonable allocation of nodes. Then, the 467 value of $z$ should be increased as much as possible to provide 468 good localization accuracy to the system. However, maximize 469 only the average does not seems fair enough, since a good 470 system should provide a certain level of accuracy homoge471 neously among the target area. So we defined the objective 472 function as difference between the term $z$ and the signal space 473 Euclidean variance

$$
Z=z-\sqrt{\sum_{l \in L}\left(\sum_{s \in D_{l}} \frac{E(l, s)}{\left|D_{l}\right|}\right)^{2}} .
$$

475 Maximizing the objective function $Z$, the intention is to 476 provide as many target location as possible with a high sig477 nal space Euclidean distance with respect to the surrounding 478 locations.
As we have previously introduced, we represent with $L$ the 479 entire set of location to be covered, while with $J$ the set of pos- 480 sible positions where nodes can be placed. By default, $L=J{ }_{481}$ and nodes can be positioned everywhere; however, its possi- ${ }_{482}$ ble to restrict the $J$ set only to specific candidate points, that ${ }^{483}$ represent for example power outlets or Ethernet sockets. The ${ }_{484}$ problem of find a near-optimal set $N$ of nodes $n_{j t}$ (each one ${ }_{485}$ located in $j$ and having a type $t$ ) with a coverage rate $f(N)$ that ${ }_{486}$ satisfies the target coverage, can be formalized as follows: ${ }_{487}$

$$
\begin{aligned}
& \max Z=z-\sqrt{\sum_{l \in L}\left(\sum_{s \in D_{l}} \frac{E(l, s)}{\left|D_{l}\right|}\right)^{2}} \\
& f(N) \geq \operatorname{target} \\
& \sum_{t \in T} x_{j t} \leq 1 \quad \forall j \in J \\
& x_{j t}=1 \quad \Longleftrightarrow n_{j t} \in N \\
& f(N)=|L| / \sum_{l \in L} y_{l} \\
& \left\{\begin{array}{l}
y_{l} \leq \sum_{j \in J} \sum_{t \in T} a_{l j t} x_{j t} \quad \forall l \in L \text { (single) } \\
2 y_{l} \leq \sum_{j \in J} \sum_{t \in T} a_{l j t} x_{j t} \quad \forall l \in L \text { (fingerprinting) } \\
3 y_{l} \leq \sum_{j \in J} \sum_{t \in T} a_{l j t} x_{j t} \quad \forall l \in L \quad \text { (trilateration). }
\end{array}\right.
\end{aligned}
$$

The decision variable $x_{j t}=1$ represents the allocation of ${ }_{494}$ a node of type $t$ in location $j ; a_{l j t}$ is equal to 1 if location $l{ }_{495}$ can be reached by a node of type $t$ placed in $j$, and $a_{l j t}=0 \quad{ }_{496}$ otherwise. $y_{l}=1$ if location $l$ is covered, $y_{l}=0$ otherwise. 497 The constraint (10) fixes to one the maximum number of nodes 498 that can be located in each site.

\section{A. Greedy Procedure}

500

The positioning algorithm starts with a Greedy procedure 501 with the purpose of find a reasonable number of reference ${ }_{502}$ nodes, for both coverage and localization accuracy. The pro- 503 cedure generate a first solution $N$ positioning a set of $k=|N|{ }_{504}$ nodes, each one with a type $t \in T$. For all three coverage tech- 505 niques, the reward $b_{l}$ is weighted with the cost of the current 506 node $n^{*}$ selected for the coverage

$$
w_{l}=\frac{b_{l}}{c_{t}} ; \quad\left\{n^{*}=n_{j t} \wedge \operatorname{distance}(j, l) \leq r_{t}\right\} .
$$

The weighted reward $w_{l}$ will be used by the Greedy algorithm 509 so that on equal covered area, the cheapest node type has 510 the priority over the others. We denote as $L_{j t}$ the subset of ${ }_{511}$ locations that are reachable by a reference node $n$ of type $t{ }_{512}$ placed at location $j$. At each iteration, the algorithm places 513 a node $n$ of type $t^{*}$ at position $j^{*}$ that covers the subset of ${ }_{514}$ locations $L_{j^{*}} t^{*}$ with the maximum reward. The term

515

$$
1-\frac{k_{l}}{k-\text { coverage }}
$$

is used to prioritize the covering of locations with a 517 lower "temporary" $k$-coverage (called $k_{l}$ ) with respect to the 518 $k$-coverage required by the current techniques. In this way, 519 Greedy procedure tends to avoid the placement of nodes very 520 


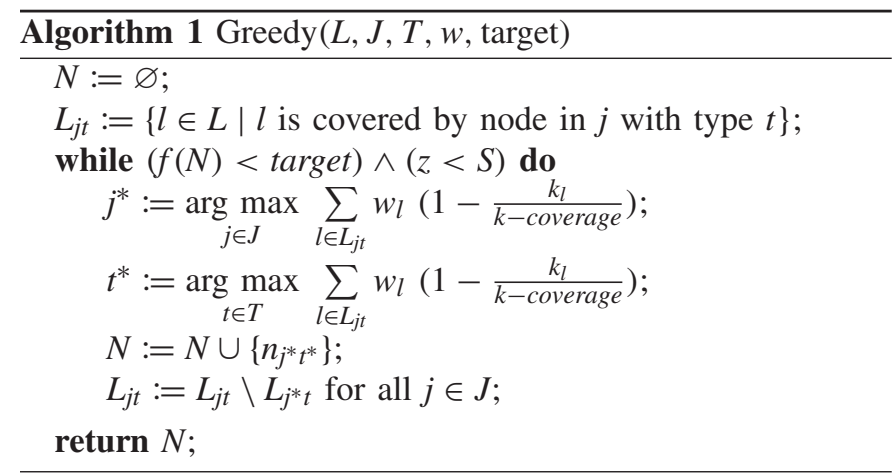

521 close to one other which can lead, especially for trilateration 522 systems, to poor localization accuracy. It is important to notice 523 that the purpose of the Greedy procedure is to find a reasonable 524 number of nodes for the localization service. The starting posi525 tioning is made on a best-effort basis, that will be improved 526 by the successive VNS. After a node allocation, all subsets ${ }_{527} L_{j t}$ are updated according to the coverage technique. In trilat528 eration for example, a location $l$ is removed from $L_{j t}$ only if 529 there exist, other than the current $n_{j^{*}} t^{*}$, other two nodes that 530 are already covering $l$.

${ }_{531}$ The Greedy procedure ends when the target coverage is sat532 isfied, and when the average signal space Euclidean distance 5 5з $z$ reaches the threshold $S$. In our implementation we set the 534 threshold $S=4.5$ that has been proven to be the average 535 Euclidean distance for which the positioning error is limited 536 to $2 \mathrm{~m}$ [5]. How we will see in Section VII, the Greedy proce${ }_{537}$ dure is able to provide an average Euclidean distance not so far 538 from the final best known. However, thanks to the low com539 plexity of the Greedy procedure, additional time can be used 540 to improve the solution. In addition, the Euclidean distance 541 variance will be strongly improved.

\section{B. Variable Neighborhood Search}

${ }_{543}$ The method called VNS has been used to improve the solu544 tion coming from the Greedy procedure. The VNS approach 545 empowers the classical local search framework with a restart 546 mechanism that extends the search after a local optimum 547 has been achieved by generating new starting solutions in 548 progressively enlarged neighborhoods of the current best 549 known solution. The key elements of the VNS (reported in 550 Algorithm 2) are a starting solution $N$ with a hierarchy of 551 size-increasing neighborhoods, and a local search procedure, 552 i.e., the criterion to select the incumbent solution from the 553 neighborhood. These components are used to restart the search ${ }_{554}$ every time that the procedure reaches a local optimum. Fig. 8 555 shows an overview of the VNS process. A first local search 556 procedure is applied to the solution produced by the Greedy 557 procedure. At each iteration, the shaking procedure is used 558 to generate a new starting solution, which is then improved 559 by the execution of the local search. The shaking procedure 560 perturbs $s$ node allocations of the current solution $N^{*}$ replac561 ing them with $s$ unused nodes. The behavior of the shaking 562 parameter $s$, that depends on the result of the local search, is 563 explained in Fig. 9. The parameter $s$ starts from a minimum

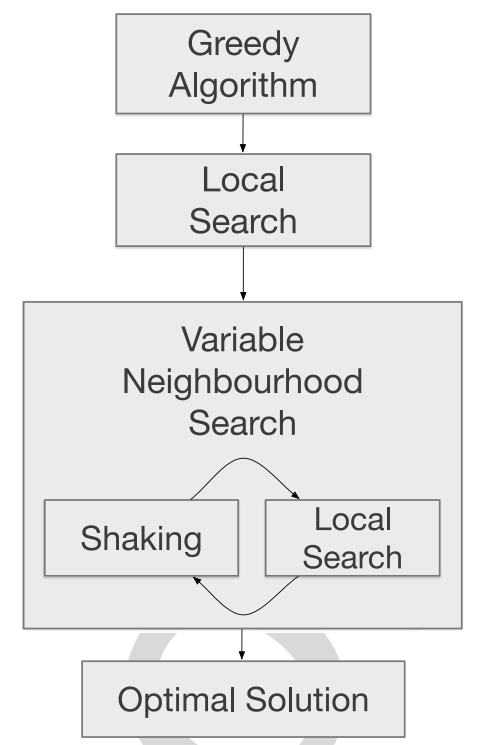

Fig. 8. Location algorithm. The solution found by the Greedy algorithm is improved applying iteratively a Local Search for an optimal solution and a Shaking procedure that perturbs the current solution.

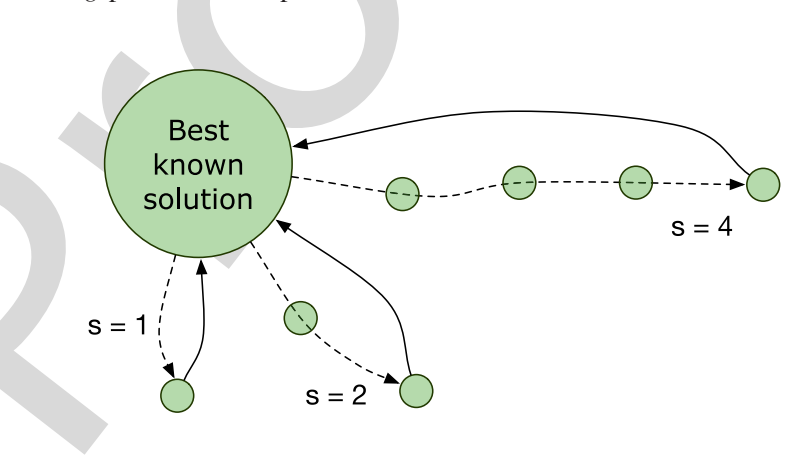

Intensification

Diversification

Fig. 9. Shaking procedure: the parameter $s$ is increased when the solution does not improve (dashed line) and restarts when a new optimum is found (continuous line).

value $s_{\min }$ (in the example $s_{\min }=1$ ) and every time that the 564 local search does not improve the best known solution, $s$ is 565 increased by 1 . Differently, when the local search succeeds, 566 the best solution $N^{*}$ is updated and $s$ goes back to $s_{\min }$. $\quad 567$

The purpose of the shaking procedure is to first explore 568 new starting solutions that are more similar to the best known 569 result, so that the search is intensified in a promising neigh- 570 borhood of the entire domain. If these local searches fail, the 571 shaking procedure moves the search from intensification to 572 diversification, generating starting solutions that are more and 573 more different from the incumbent one. Whenever a new best 574 solution is found, the shaking procedure comes back to $s_{\min }$, to 575 intensify the search near the just updated $N^{*}$. In principle, the 576 shaking parameter $s$ can be increased until $k=\left|N^{*}\right|$, changing 577 all the node allocations. However, we experimented running 578 different configurations that excessively moving away from 579 the best known solution can be unproductive, causing a use- 580 less waste of computational time. We have fixed a reasonable 581 value of $s_{\max }=\lfloor(2 / 3) k\rfloor$. 


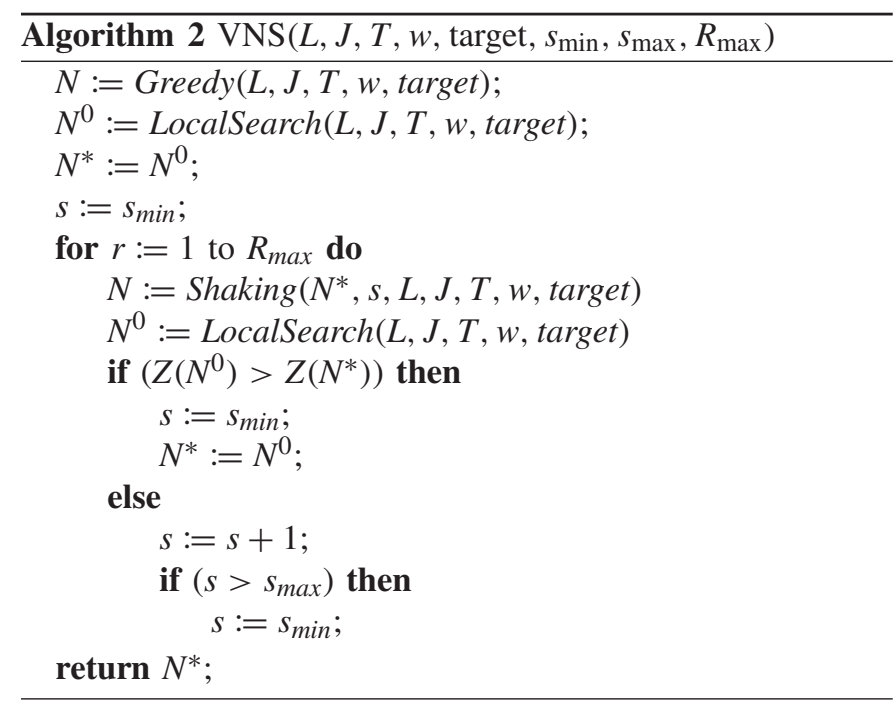

583 The VNS algorithm terminates when the total number of 584 restarts reaches a given value $R_{\max }$.

585 As we have said, the local search is the heuristic that 586 proceeds from an initial solution to its neighborhood by a 587 sequence of local changes, trying to improve each time the 588 value of the objective function until a local optimum is found. 589 The neighborhood of the adopted approach is given by cyclic 590 sequences of moves, where each move consists in locating a 591 new node, removing a node or changing the type of the node. 592 A cyclic move is considered feasible only if the new covering ${ }_{593}$ rate respects the target coverage, and the total cost of the solu594 tion does not increase. Of course, each site must continue to 595 hosts at maximum one node [constraint (10)]. A cyclic move 596 can be visualized on a graph $G=(N, A)$, where each node of 597 the graph is a possible allocation of a hardware node. Each 598 node of the graph is characterized by a location $j$, and a state 599 that indicates if the node is active or inactive. A node $n_{j t}$ cur600 rently allocated in location $j$, is represented on the graph with 601 an active node $n_{j}$, labeled with its hardware type $t$. Note that 602 index $t$ does not appear because at most one type can be active 603 in each node, and the type is specified by the label. Inactive 604 nodes are instead left unlabeled. An arc $\left(n_{j}, n_{k}\right)$ can represent 605 the following.

606 1) The allocation of a hardware node in site $j$, if $n_{j}$ is inactive and $n_{k}$ is active.

2) The removal of a hardware node in site $j$, if $n_{j}$ is active and $n_{k}$ is inactive.

610

611

3) An hardware node $n_{j}$ changing its hardware type, if both nodes are active.

612 In both 1) and 2), the new node takes the hardware type of 613 the head label ( $t$ of $n_{k}$ ). A cyclic exchange corresponds to 614 a directed cycle on the improvement graph, as depicted in 615 Fig. 10. Each move, and so each $\operatorname{arc}\left(n_{j}, n_{k}\right)$, determines a vari616 ation $\delta Z$ in the value of the objective function $Z$. The purpose 617 is to represent a group of moves so that a cyclic exchange rep618 resents an increase in the current objective function. However, 619 the total variation $\delta Z$ is non additive with respect to the 620 sequence of $\delta Z$ values coming from single moves. This is ${ }_{621}$ caused by the interdependence between different hardware

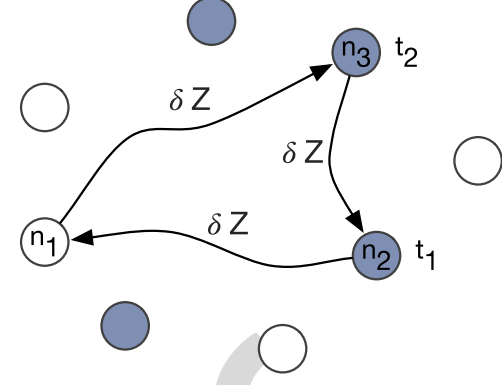

Fig. 10. Improvement graph: colored nodes represent current allocations, while empty nodes are possible allocations. All active nodes are labeled with their corresponding type. Each arc is a change (move) on the allocations.

nodes with overlapping covering regions, that lead to nonaddi- 622 tive moves. To overcome this drawback, every cycle has been ${ }^{623}$ evaluated using an own temporary function $Z^{\prime}$ updated step by ${ }_{624}$ step from the end of the path to its starting node. In this way, ${ }_{625}$ all the cycles with a positive total weight bring improvements 626 on the starting solution.

The search for the cyclic exchange with maximum weight ${ }_{628}$ is performed with exhaustive breadth-first exploration of the 629 paths of graph $G$.

\section{EXPERIMENTAL RESULTS}

631

Presented experimental results are initially focused on the 632 usability of the tool, testing the ability to provide a solution ${ }_{63}$ in a reasonable time. Then, the performances of the model ${ }_{634}$ have been evaluated, in terms of localization accuracy through ${ }_{635}$ realistic indoor environment experiments, and in terms of cost- ${ }_{336}$ effectiveness of the suggested deployments.

\section{A. Computational Experience}

638

The tool has been evaluated running several different config- 639 urations. Every test reported in this section has been executed 640 with a spatial resolution of the floor plan equal to $1 \mathrm{~m}$. A first ${ }_{641}$ analysis can be done on the execution times of the proposed ${ }_{642}$ solution. Although the execution time can be tuned by the ${ }_{643}$ parameter $R_{\max }$, which represents the maximum number of 644 restarts of the VNS algorithm, an idea on the order of mag- 645 nitude is given by Fig. 11, where the time is represented as 646 a function of the floor-plan dimension. In the given example, ${ }_{647}$ $R_{\max }$ has been fixed to 20 restarts, the target coverage equals 648 to $95 \%$ of the total area, a single node type available with a 649 range of $8 \mathrm{~m}$, covering floor-plans with rectangular areas. The 650 graph shows that for single coverage and fingerprinting the 651 processing time grows approximately linearly with the floor 652 plan area.

653

A numeric comparison of the same tests is reported in 654 Table IV, where execution times are reported in seconds for 655 increasing floor plans. For single coverage, the execution time 656 is low even for areas of 3000 squared meters. For trilatera- 657 tion and fingerprinting, the execution times become high from 658 floor-plan of $2500 \mathrm{~m}^{2}$. However, the tests represent a bad case 659 in which the map dimension is very large while the node range 660 available and the spatial resolution are small (respectively, 661 


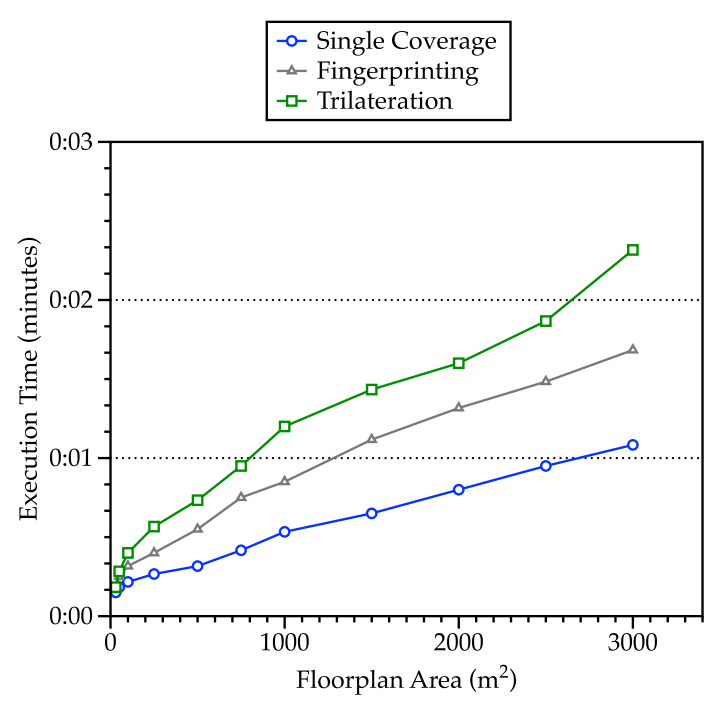

Fig. 11. Execution time of the tool with floor plans of different areas, for each covering technique $\left(R_{\max }=20\right.$, target $=95 \%$, and $\left.r_{t}=8\right)$.

TABLE IV

EXECUTION TIME OF THE TOOL FOR INCREASING FLOOR Plan AREAS $\left(R_{\max }=20\right.$, target $=95 \%$, AND $\left.r_{t}=8\right)$

\begin{tabular}{|c|c|c|c|}
\hline \multirow{2}{*}{$\begin{array}{c}\text { Floor Plan } \\
\text { Area }\left(m^{2}\right)\end{array}$} & \multicolumn{3}{|c|}{ Execution Time (s) } \\
\cline { 2 - 4 } & Single & Fingerprinting & Trilateration \\
\hline 30 & 9.07 & 10.53 & 11.49 \\
50 & 11.31 & 15.10 & 17.67 \\
100 & 13.05 & 19.41 & 24.22 \\
250 & 16.57 & 24.89 & 34.16 \\
500 & 19.18 & 33.48 & 44.66 \\
750 & 25.43 & 45.32 & 57.94 \\
1000 & 32.19 & 51.68 & 72.83 \\
1500 & 39.37 & 67.12 & 86.27 \\
2000 & 48.30 & 79.49 & 96.11 \\
2500 & 57.11 & 89.47 & 112.34 \\
3000 & 65.41 & 101.24 & 139.18 \\
\hline
\end{tabular}

6628 and $1 \mathrm{~m}$ ). Increasing the range or the resolution, the instance 663 of the problem decrease, resulting in faster executions.

${ }_{664}$ A key aspect that characterizes the goodness of the 665 proposed approach is the improvement of the objective func666 tion achieved by the VNS algorithm with respect to the first ${ }_{667}$ Greedy configuration. For this test we have run the tool sev668 eral times with a floor-plan area of $2500 \mathrm{~m}^{2}$ and a node range 669 of $12 \mathrm{~m}$. The number of reference nodes allocated is deter670 mined by the Greedy procedure and increase with $S$, while 671 the number of VNS restarts $R_{\max }$ has been fixed to 35 .

672 In Fig. 12, we reported the value of $z$, i.e., the average signal 673 space Euclidean distance obtained with the first Greedy exe674 cution, compared with the $z$ value after the VNS optimization. 675 The graph reports the $z$ values as a function of the threshold $S$, 676 described in Section VI-A as the minimum value of average 677 signal space Euclidean distance $(z)$ required during the Greedy 678 procedure. The graph shows that moving the threshold within

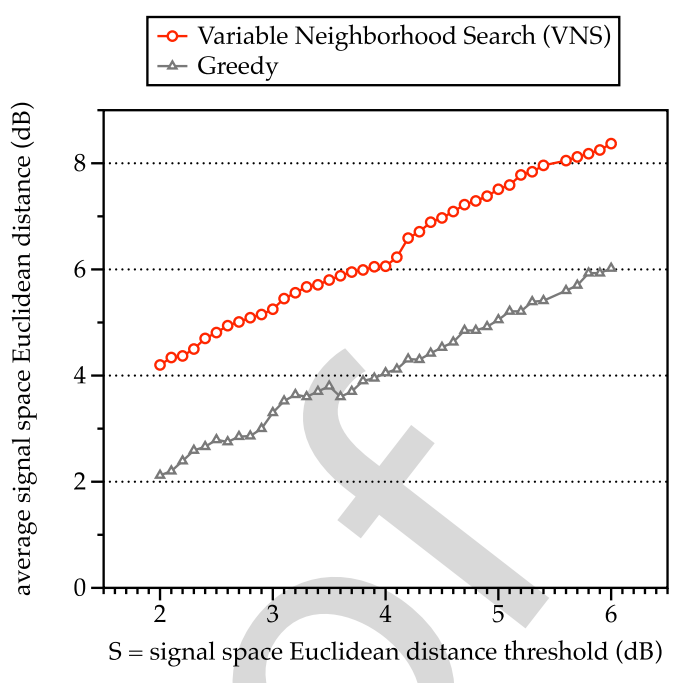

Fig. 12. Average signal space Euclidean distance $(z)$ obtained with the Greedy execution and compared with the $z$ value after the VNS optimization. $z$ values expressed as a function of the threshold $S$. Floor-plan area $=2500 \mathrm{~m}^{2}$, $R_{\max }=20$, target $=100 \%$, and $r_{t}=12$.

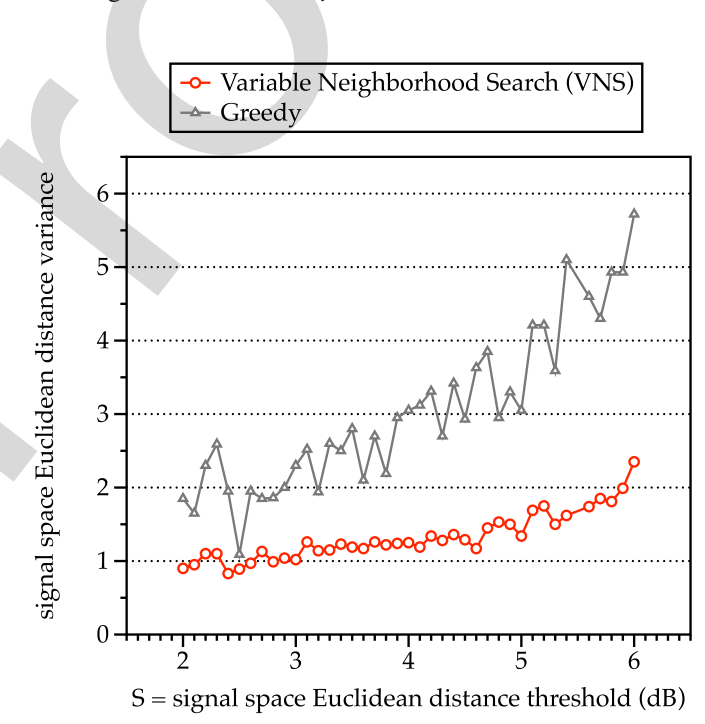

Fig. 13. Signal space Euclidean distance variance obtained with the Greedy execution and compared with the $z$ value after the VNS optimization. Values expressed as a function of the threshold $S$. Floor-plan area $=2500 \mathrm{~m}^{2}, R_{\max }=$ 20 , target $=100 \%$, and $r_{t}=12$.

the range $(2,6) \mathrm{dB}$ the VNS is able to improve the $z$ value con- 679 stantly around $2 \mathrm{~dB}$. Although the VNS improvement is not 680 astonishing for what regard the average value, Fig. 13 shows 681 that the variance is strongly improved. This has been achieved 682 moving from the objective function $z$ used in Greedy proce- 683 dure to the $Z$ function of the VNS. The $Z$ objective function 684 has in fact the purpose to provide as many target location as 685 possible with a high signal space Euclidean distance w.r.t. the 686 surrounding locations.

\section{B. Experimental Setup and Accuracy Evaluation}

688

The proposed tool was evaluated using data collected from 689 a real-world environment, the NECST Lab, located at the 690 basement of DEIB Department at the Politecnico di Milano. 691 


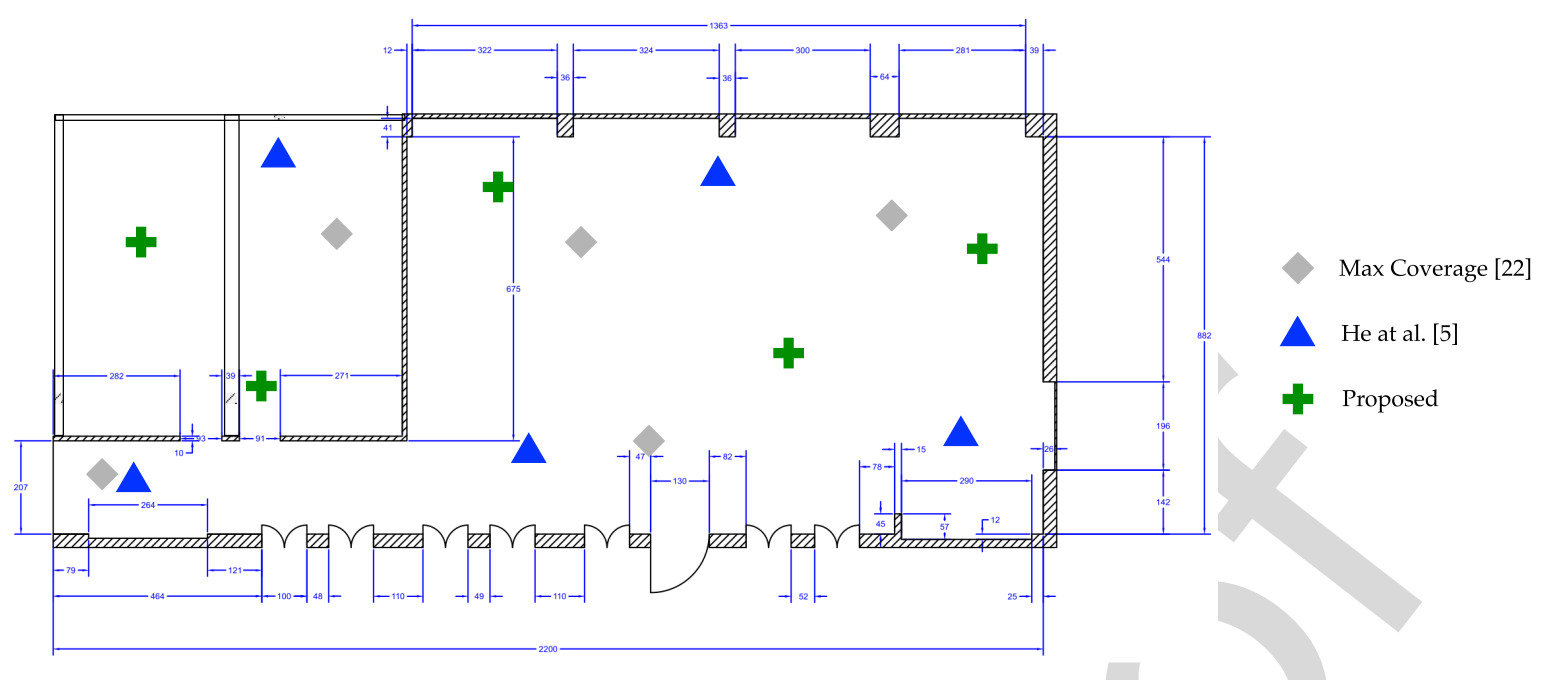

Fig. 14. NECST Laboratory floor-plan, located at the basement of DEIB Department at the Politecnico di Milano. Each allocation corresponds to a BLE beacon with a range of $7 \mathrm{~m}$. Green crosses indicates allocations provided by our algorithm, gray rhombus represent allocations from [5] while blue triangle positions have been computed maximizing the coverage [22].

692 The dimension of the test-bed is 198 squared meters $(9 \times 22 \mathrm{~m})$. ${ }_{693}$ We collected BLE signal data coming from BLE beacons with 694 a coverage radius of $7 \mathrm{~m}$. Signal data has been collected 695 using a Nexus 5 smartphone running Android 6.0.1. First, the 696 NECST Laboratory floor-plan has been designed using our 697 tool, obtaining the optimal number of beacons $(|N|=5)$ and 698 their allocation for fingerprinting localization. $R_{\max }$ has been 699 fixed to 20 restarts, the target coverage equals to $100 \%$ of the 700 total area, a single node type available with a range of $7 \mathrm{~m}$, and 701 the threshold $S=4,5$. We collected 40 training samples for 702 the localization algorithm using the obtained allocation. Then, 703 the test samples were collected at distinct positions changing 704 the phone orientation and the way in which user was keeping 705 it, for example by hand or in a pocket. For the entire duration 706 of training and test phase, the number of occupants and their 707 enabled wireless devices has changed, from a minimum of 3 to 708 a maximum of 17 people. This variation affects the accuracy 709 performances, but at the same time contributes in obtaining 710 realistic results. The training and test phase has been repeated 711 with two configurations coming from different allocation algo712 rithms: maximization of the coverage [22] and the allocation 713 algorithm proposed by He et al. [5]. For these two algorithms, 714 the number of employed nodes has been fixed to 5. KNN with $715 K=3$ has been employed as the fingerprinting algorithm.

${ }_{716}$ A first result is shown in Fig. 15. The cumulative error 717 distribution function shows that from $1.5 \mathrm{~m}$ our approach per718 forms better. Under $1.5 \mathrm{~m}$, He et al. [5] approach performs 719 better, but the difference in accuracy is marginal.

720 Fig. 16 shows the mean positioning accuracy divided into 721 different error ranges: $(0,0.5],(0.5,1],(1,1.5],(1.5,2]$, $722(2,2.5],(2.5,3],(3,3.5]$, and $(3.5,4]$. It is possible to notice 723 that the majority of the localization errors appears within the $724(1.5,2] \mathrm{m}$. The test-bed floor-plan, composed by three rooms, 725 has been reported in Fig. 14. Green crosses indicates allo726 cations provided by our algorithm, gray rhombus represent 727 allocations from [5] while blue triangle positions have been 728 computed maximizing the coverage [22].

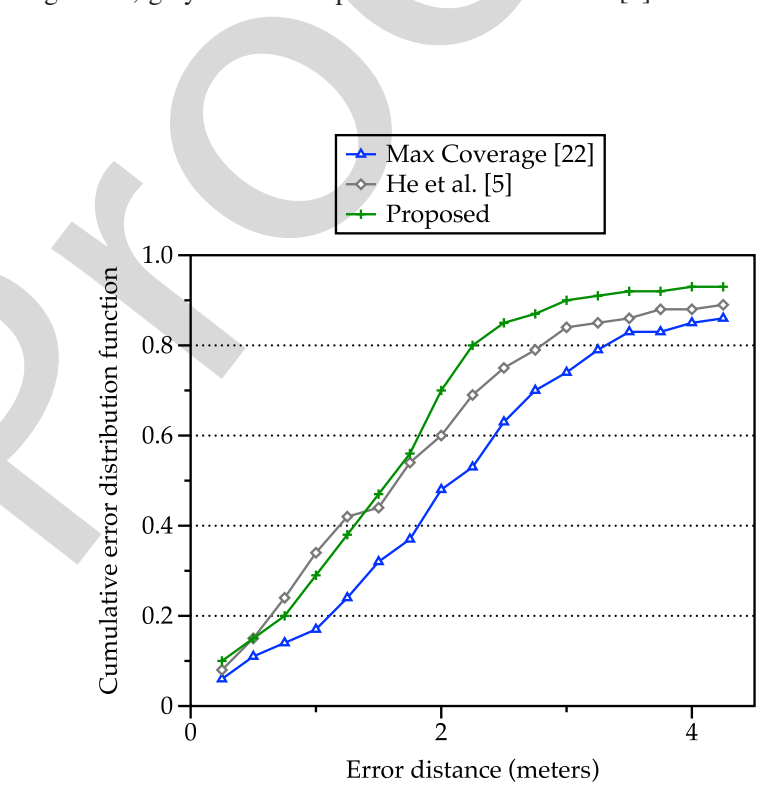

Fig. 15. Cumulative error distribution function experienced by our approach ad compared with two different solutions from the state-of-the-art.

\section{Cost-Effectiveness Analysis}

A feature of our tool interesting for testing is the possibility 730 to obtain solutions from mixed node types, with different char- 731 acteristics and costs. In particular, given two types $t_{1}$ and $t_{2}{ }_{732}$ characterized by two ranges $r_{i}$, and two costs $c_{i}$, it is possible ${ }_{733}$ to compare the total cost of a homogeneous solution with the 734 cost of a mixed solution. Given a baseline type of node with 735 a range $r_{1}=8 \mathrm{~m}$ and a cost of $c_{1}=60 \$$, we can assume ${ }_{736}$ the presence on the market of a second type of hardware, with 737 the half of the range distance $\left(r_{2}=4 \mathrm{~m}\right)$. The area covered ${ }_{738}$ by $t_{1}\left(\approx 200 \mathrm{~m}^{2}\right)$ is four times bigger than the coverage of $t_{2}{ }_{739}$ $\left(\approx 50 \mathrm{~m}^{2}\right)$. In order to obtain a fair test, the cost of $t_{2}$ should 740 be $c_{2} \geq c_{1} / 4$, and so we set $c_{2}=20 \$$. This test has been 741 performed with a target coverage of $95 \%$ on a rectangular map 742 of $1000 \mathrm{~m}^{2}$.

From Table V, it is possible to observe that, although hard- 744 ware nodes of type $t_{2}$ have a lower convenience in terms of 745 


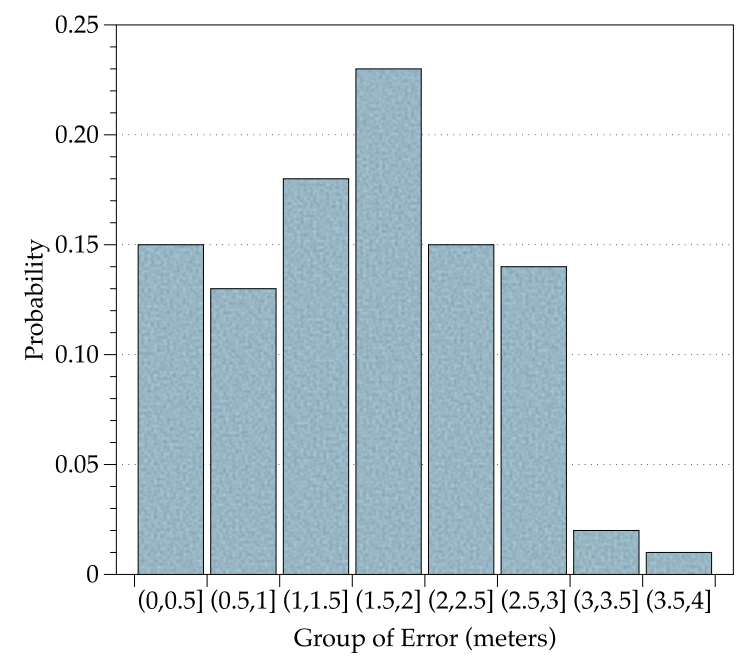

Fig. 16. Mean positioning accuracy of the proposed allocation algorithm divided into different error ranges.

TABLE V

Cost of Homogeneous and Mixed Solutions $\left(A=1000 \mathrm{~m}^{2}\right.$ target $=95 \%, r_{1}=8 \mathrm{~m}, r_{2}=4 \mathrm{~m}, c_{1}=60 \$$, AND $c_{2}=20 \$$ )

\begin{tabular}{|c|c|c|c|}
\hline \multirow{2}{*}{ Node types } & \multicolumn{3}{|c|}{ Solution Costs (in \$) } \\
\cline { 2 - 4 } & Single & Trilateration & Fingerprinting \\
\hline$T=\left\{t_{1}\right\}$ & 480 & 1440 & 840 \\
$T=\left\{t_{2}\right\}$ & 500 & 1620 & 880 \\
$T=\left\{t_{1}, t_{2}\right\}$ & 440 & 1280 & 760 \\
\hline
\end{tabular}

746 (area/price) ( $t_{1}$ outperform $t_{2}$ in homogeneous solutions), the 747 mixed strategy can use the smaller range nodes to reduce the 748 total cost. This because less powerful nodes of type $t_{2}$ are 749 employed to cover small portions of the floor-plan, like corners 750 or small regions left uncovered by the larger range nodes.

751 The amount of saving in the total cost of the mixed solu752 tion does not depend only on the nodes range and price, but 753 also on the irregularity of the floor plan perimeter. A distin754 guish feature of the proposed tool respect to other works is 755 the possibility to cover spaces that are not necessarily rectan756 gular or squared. The level of irregularity of a floor plan can 757 be identified by the minimum number of rectangles that com758 pose the shape. In Fig. 17 for example, the index of the floor 759 plan irregularity is $I=4$. We experimented the behavior of 760 the tool increasing the level of irregularity, while maintaining 761 a constant total area of $1000 \mathrm{~m}^{2}$. The test has been done with 762 the same nodes configuration used in Table V (homogeneous ${ }_{763} T=t_{1}$, mixed $\left.T=t_{1}, t_{2}\right)$. The results shown in Table VI 764 proven that increasing the floor-plan irregularity, the cost dif765 ference between homogeneous and mixed solution becomes 766 higher. This is caused by the increasing number of corners in 767 the map, that can be covered with less powerful nodes.

768 In conclusion, experimental results show that for most of 769 the problem instances, a solution can be obtained in reason770 able execution times. Depending on the available hardware 771 types, homogeneous solutions could be improved with the 772 employment of different type of nodes.

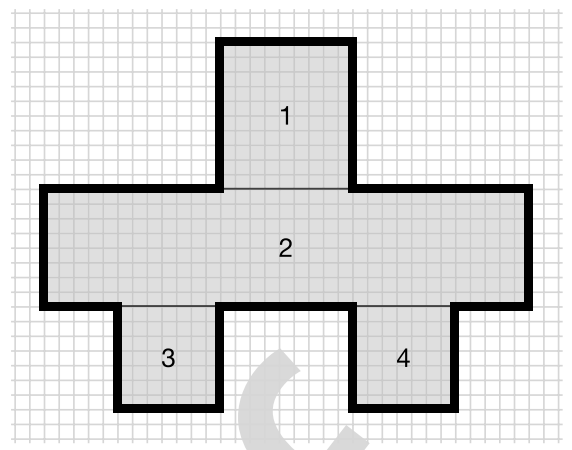

Fig. 17. Irregularity of the floor-plan perimeter summarized by the minimum number of rectangles.

TABLE VI

Cost DifFEREnCES (IN \$) BETWEen HomogeneOUS AND MiXed SOLUTION INCREASING THE FLOOR PLAN IRREGULARITY (AREA FIXED TO $1000 \mathrm{~m}^{2}$ )

\begin{tabular}{|c|cc|cc|cc|}
\hline \multirow{2}{*}{$I$} & \multicolumn{2}{|c|}{ Single } & \multicolumn{2}{c|}{ Trilater. } & \multicolumn{2}{c|}{ Fingerprint. } \\
\cline { 2 - 7 } & homog. & mixed & homog. & mixed & homog. & mixed \\
\hline 1 & 480 & 440 & 1440 & 1280 & 840 & 760 \\
2 & 480 & 440 & 1500 & 1320 & 840 & 780 \\
4 & 600 & 500 & 1560 & 1380 & 900 & 820 \\
8 & 720 & 580 & 1680 & 1480 & 1200 & 920 \\
\hline
\end{tabular}

\section{CONCLUSION}

773

In this paper, we tried to explain the challenges faced by 774 designers during the installation of smart building systems that 775 require the positioning of several hardware nodes. A com- 776 mon limitation of existing models is the lack of a convenient 777 way to specify geometric information of the indoor map. This 778 also leads to the employment of less accurate general models 779 for signal propagation, instead of site-specific models. The 780 design phase is made more difficult by the availability on 781 the market of different hardware nodes, with different power 782 transmissions and costs.

For these reasons we propose an integrated tool for both 784 floor plan specification and node positioning, developed within 785 an open-source CAD environment extensible through plug-ins. 786 The tool is able to provide a near-optimal solution of node 787 allocations, possibly with mixed types, with the aim to reduce 788 the installation costs. The results suggest that, for most of 789 the problem instances, a solution can be obtained in a rea- 790 sonable execution time. Depending on the available hardware 791 types, total cost of the solution could be improved moving 792 from homogeneous to mixed type allocation. 793

A limitation of the proposed approach resides in the prop- 794 agation model used to compute near-optimal solutions for 795 localization systems. The model implemented is site-specific, 796 and take in consideration walls for LOS and NLOS prop- 797 agations. However, the approach do not consider refraction 798 or diffraction effects. Another limitation is the inability of 799 the system to model the signal propagation between differ- 800 ent floors of the building, managing each level independently. 801 For future work, we plan to improve the system with an 802 indoor signal propagation model able to consider refraction 803 and diffraction effects of the indoor environment like walls 804 and floors. In addition, we will try to apply the model to 805 
806 3-D designing tools, becoming suitable also for multifloor 807 environments.

\section{REFERENCES}

[1] C.-A. Roulet, "Indoor environment quality in buildings and its impact on outdoor environment," Energy Build., vol. 33, no. 3, pp. 183-191, 2001.

[2] V. L. Erickson, M. Á. Carreira-Perpiñán, and A. E. Cerpa, "OBSERVE: Occupancy-based system for efficient reduction of HVAC energy," in Proc. 10th ACM/IEEE Int. Conf. Inf. Process. Sensor Netw., Chicago, IL, USA, 2011, pp. 258-269. [Online]. Available: http://ACMBuildSys2015.com

[3] B. Balaji, J. Xu, A. Nwokafor, R. Gupta, and Y. Agarwal, "Sentinel: Occupancy based HVAC actuation using existing WiFi infrastructure within commercial buildings," in Proc. 11th ACM Conf. Embedded Netw. Sensor Syst., Rome, Italy, 2013, p. 17.

4] Y. Zhao, H. Zhou, and M. Li, "Indoor access points location optimization using differential evolution," in Proc. Int. Conf. Comput. Sci. Softw. Eng., Wuhan, China, 2008, pp. 382-385. [Online]. Available: http:// ieeexplore.ieee.org/lpdocs/epic03/wrapper.htm?arnumber $=4721767$

[5] Y. He, W. Meng, L. Ma, and Z. Deng, "Rapid deployment of APs in WLAN indoor positioning system," in Proc. 6th Int. ICST Conf. Commun. Netw. China (CHINACOM), Harbin, China, 2011, pp. 268-273.

6] S.-H. Fang and T.-N. Lin, "A novel access point placement approach for WLAN-based location systems," in Proc. IEEE Wireless Commun. Netw. Conf. (WCNC), Sydney, NSW, Australia, 2010, pp. 1-4.

7] ArchiCAD—The Architectural BIM CAD Software. [Online]. Available: http://www.graphisoft.com/archicad/

[8] J. P. Zhang and Z. Z. Hu, "BIM-and 4D-based integrated solution of analysis and management for conflicts and structural safety problems during construction: 1. Principles and methodologies," Autom. Construct., vol. 20, no. 2, pp. 167-180, 2011.

[9] Y. G. Xu, C. Qian, W.-P. Sung, J. C. M. Kao, and R. Chen, "Lean cost analysis based on BIM modeling for construction project," Front. Mech. Eng. Mater. Eng. II, vols. 457-458, pp. 1444-1447, 2014. [Online]. Available: http://www.scientific.net/AMM.457-458.1444.pdf

42 [10] M. S. Daskin, "A maximum expected covering location model: Formulation, properties and heuristic solution," Transp. Sci., vol. 17, no. 1, pp. 48-70, 1983. [Online]. Available: http://www.scopus.com/ inward/record.url?eid=2-s2.0-0020707868 $\{\&\}$ partnerID=tZOtx3y 1

846 [11] M. S. Daskin and E. H. Stern, "A hierarchical objective set covering model for emergency medical service vehicle deployment," Transp. Sci., vol. 15, no. 2, pp. 137-152, 1981. [Online]. Available: http://www.scopus.com/inward/record.url?eid=2-s2.0-0019565514\{\&\} partnerID=tZOtx3y 1

51 [12] V. T. Quang and T. Miyoshi, "An algorithm for sensing coverage problem in wireless sensor networks," in Proc. IEEE Sarnoff Symp., Princeton, NJ, USA, 2008, pp. 1-5.

54 [13] A. M.-C. So and Y. Ye, "On solving coverage problems in a wireless sensor network using Voronoi diagrams," in Lecture Notes in Computer Science (Including Subseries Lecture Notes in Artificial Intelligence and Lecture Notes in Bioinformatics) (LNCS 3828). Heidelberg, Germany: Springer, 2005, pp. 584-593.

9 [14] T. Andersson. (2014). Bluetooth Low Energy and Smartphones for Proximity-Based Automatic Door Locks. [Online]. Available: http://www.diva-portal.org/smash/record.jsf?pid=diva2:723899\{\&\} dswid=-9677

[15] A. S. Paul et al., "MobileRF: A robust device-free tracking system based on a hybrid neural network HMM classifier," in Proc. ACM Int. Joint Conf. Pervasive Ubiquitous Comput., Seattle, WA, USA, 2014, pp. 159-170. [Online]. Available: http://doi.acm.org/10.1145/2632048.2632097

[16] V. L. Erickson, S. Achleitner, and A. E. Cerpa, "POEM: Power-efficient occupancy-based energy management system," in Proc. 12th Int. Conf. Inf. Process. Sensor Netw., Philadelphia, PA, USA, 2013, pp. 203-216.

[17] A. Beltran, V. V. L. Erickson, and A. E. A. Cerpa, "ThermoSense: Occupancy thermal based sensing for HVAC control," in Proc. 5th ACM Workshop Embedded Syst. Energy Efficient Build., Rome, Italy, 2013, pp. 1-8. [Online]. Available: http://doi.acm.org/10.1145/2528282.252 8301\$ndelimiter”026E30F\$nhttp://dl.acm.org/citation.cfm?id=2528301

876 [18] Y. Zhao, A. LaMarca, and J. R. Smith, "A battery-free object localization and motion sensing platform," in Proc. ACM Int. Joint Conf. Pervasive Ubiquitous Comput. UbiComp Adjunct, Seattle, WA, USA, 2014, pp. 255-259. [Online]. Available: http://dx.doi.org/ 10.1145/2632048.2632078\$ndelimiter"026E30F\$nhttp://dl.acm.org/ citation.cfm?doid $=2632048.2632078$

[19] A. Corna, L. Fontana, A. A. Nacci, and D. Sciuto, "Occupancy detection 882 via iBeacon on android devices for smart building management," in 883 Proc. Des. Autom. Test Eur. Conf. Exhibit., Grenoble, France, 2015, 884 pp. 629-632.

[20] P. Kyösti et al., "IST-4-027756 WINNER II D1. 1.2 V1. 2 WINNER 886 II channel models.pdf," Projectscelticinitiativeorg, vol. 1, no. 82, 887 p. 82, 2008. [Online]. Available: http://projects.celtic-initiative.org/ 888 winner+/WINNER2-Deliverables/D1.1.2v1.2.pdf

[21] F. Colombo, R. Cordone, and G. Lulli, "The multimode covering loca- 890 tion problem," Comput. Oper. Res., vol. 67, pp. 25-33, Mar. 2016. 891 [Online]. Available: http://dx.doi.org/10.1016/j.cor.2015.09.003 892

[22] M. Kouakou, S. Yamamoto, K. Yasumoto, and M. Ito "Cost-efficient 893 deployment for full-coverage and connectivity in indoor 3D WSNs," in 894 Proc. IPSJ Dicomo, 2010, pp. 1975-1982.

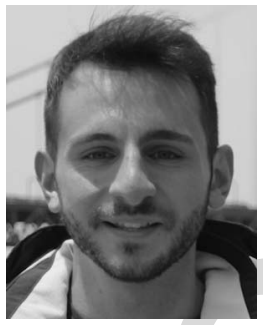

Andrea Cirigliano received the B.Sc. degree 896 in computer engineering from the Politecnico di 897 Milano, Milan, Italy, in 2013, where he is currently 898 pursuing the M.Sc. degree. 899

He joined the NECST Laboratory, Politecnico di 900 Milano, in 2015, where he is currently research- 901 ing on nonintrusive indoor localization systems and 902 occupancy detection systems. His current research 903 interests include design of smart building systems, 904 wireless indoor localization, and pervasive data 905 management.

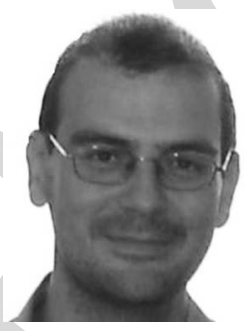

Roberto Cordone received the Dr.Eng. degree 907 in electronic engineering and the Ph.D. degree 908 in computer science and control theory from the 909 Politecnico di Milano, Milan, Italy, in 1996 and 910 2000 , respectively.

$\mathrm{He}$ is currently an Assistant Professor with the 912 Universita' degli Studi di Milano, Milan. His cur- 913 rent research interests include operations research 914 and algorithm design and analysis. $\quad 915$

Dr. Cordone is a member of the Italian 916 Association of Operations Research. $\quad 917$

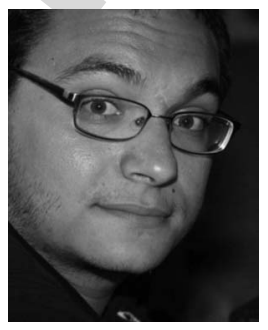

Alessandro A. Nacci received the B.Sc. and 918 M.Sc. degrees in computer engineering from the 919 Politecnico di Milano, Milan, Italy, in 2009 and 920 2012, respectively, where he is currently pursuing 921 the Ph.D. degree.

He was with EPFL, Lausanne, Switzerland. He 923 was with the Telecom Italia Joint Open Laboratory 924 S-Cube and the NECST Laboratory on the smart 925 buildings topic with the Politecnico di Milano, where 926 he has been a Research Affiliate and a Teaching 927 Assistant, since 2016. He was a Post-Doctoral 928 Research Fellow with the University of California at San Diego, San Diego, 929 CA, USA, for six months, researching at the Synergy Laboratory on the smart 930 complex buildings. In 2014, he started two companies within the Internet of 931 Things and smart building market.

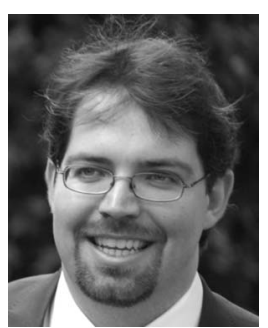

Marco Domenico Santambrogio (SM'XX) 933 AQ7 received the laurea (M.Sc. equivalent) degree in 934 computer engineering from the Politecnico di 935 Milano, Milan, Italy, in 2004, the second M.Sc. 936 degree in computer science from the University of 937 Illinois at Chicago, Chicago, IL, USA, in 2005, and 938 the $\mathrm{Ph} . \mathrm{D}$. degree in computer engineering from the 939 Politecnico di Milano, in 2008.

$\mathrm{He}$ is an Assistant Professor with the Politecnico 941 di Milano. He was a Post-Doctoral Fellow with 942 CSAIL, MIT, Cambridge, MA, USA, and has also 943 held visiting positions at the Department of Electrical Engineering and 944 Computer Science, Northwestern University, Evanston, IL, USA, in 2006945 and 2007, and Heinz Nixdorf Institute, Paderborn, Germany, in 2006. He has 946 been with the NECST Laboratory, Politecnico di Milano, where he founded 947 the Dynamic Reconfigurability in Embedded System Design project in 2004948 and the CHANGE (self-adaptive computing system) project in 2010. His 949 current research interests include reconfigurable computing, self-aware and 950 autonomic systems, hardware/software co-design, embedded systems, and 951 high performance processors and systems.

Dr. Santambrogio is a Senior Member of ACM. 


\section{AUTHOR QUERIES \\ AUTHOR PLEASE ANSWER ALL QUERIES}

PLEASE NOTE: We cannot accept new source files as corrections for your paper. If possible, please annotate the PDF proof we have sent you with your corrections and upload it via the Author Gateway. Alternatively, you may send us your corrections in list format. You may also upload revised graphics via the Author Gateway.

AQ1: Please confirm that the e-mail id for the author "A. Cirigliano" is correct as set.

AQ2: Please provide the in-text citation for "Tables I and III," "Figs. 1 and 2," and "Algorithm 1."

AQ3: Please provide the accessed date for Reference [7].

AQ4: Please provide the issue number or month for Reference [9].

AQ5: Please confirm that the edits made to References [10]-[13] and [22] are correct as set.

AQ6: Please confirm that the location and publisher information for Reference [13] is correct as set.

AQ7: Please provide the membership year for the author "M. D. Santambrogio." 


\title{
Toward Smart Building Design Automation: Extensible CAD Framework for Indoor Localization Systems Deployment
}

\author{
Andrea Cirigliano, Roberto Cordone, Alessandro A. Nacci, and \\ Marco Domenico Santambrogio, Senior Member, IEEE
}

\begin{abstract}
Over the last years, many smart buildings 2 applications, such as indoor localization or safety systems, have 3 been subject of intense research. Smart environments usually rely 4 on several hardware nodes equipped with sensors, actuators, and 5 communication functionalities. The high level of heterogeneity 6 and the lack of standardization across technologies make design 7 of such environments a very challenging task, as each instal\& lation has to be designed manually and performed ad-hoc for 9 the specific building. On the other hand, many different systems 10 show common characteristics, like the strict dependency with 11 the building floor plan, also sharing similar requirements such 12 as a nodes allocation that provides sensing coverage and nodes 13 connectivity. This paper provides a computer-aided design appli14 cation for the design of smart building systems based on the 15 installation of hardware nodes across the indoor space. The 16 tool provides a site-specific algorithm for cost-effective deploy17 ment of wireless localization systems, with the aim to maximize 18 the localization accuracy. Experimental results from real-world 19 environment show that the proposed site-specific model can 20 improve the positioning accuracy of general models from the ${ }_{21}$ state-of-the-art. The tool, available open-source, is modular and 22 extensible through plug-ins allowing to model building systems 23 with different requirements.
\end{abstract}

24 Index Terms-Indoor localization, Internet of Things, 25 performance optimization, smart buildings design automation.

26

$\mathbf{O}$ N AVERAGE, people spend approximately $70 \%$ of their time indoors [1], such as in offices, schools, and at 29 home. New indoor smart applications are being developed 3o at high rate, in both research and commercial areas cover${ }_{31}$ ing a wide range of personal and social scenarios. Smart 32 buildings are becoming a reality with the adoption of an under${ }_{33}$ lying monitoring and communication infrastructure composed 34 by access points (APs), sensor motes, cameras, and smart 35 devices integrated in a building management systems (BMSs).

Manuscript received September 14, 2016; accepted November 13, 2016. This paper was recommended by Associate Editor S. Mohanty.

A. Cirigliano, A. A. Nacci, and M. D. Santambrogio are with the Department of Electronics, Information and Bioengineering, Politecnico di Milano, 20133 Milan, Italy (e-mail: andrea.cirigliano@mail.polimi.it).

R. Cordone is with the Department of Computer Science, Universita' degli Studi di Milano, 20100 Milan, Italy.

Color versions of one or more of the figures in this paper are available online at http://ieeexplore.ieee.org.

Digital Object Identifier 10.1109/TCAD.2016.2638448
The BMS is a control system that monitors the building state 36 and operates through actuators to increase the comfort and ${ }_{37}$ safety of occupants, while managing the energy efficiency at 38 the same time.

Many smart buildings applications are based on indoor 40 localization techniques, using location information to optimize ${ }_{41}$ the environment and provide context-aware services. Indoor 42 localization systems often require the presence of wireless 43 devices such as APs, in order to let the user identify its position ${ }_{44}^{4}$ by means of a mobile device. Most smart building applica- 45 tions have been developed in order to achieve sustainability, reducing energy waste related to energy-consuming appliances like heating, ventilation, and air-conditioning (HVAC). Some examples are [2] and [3]. Smart HVAC systems usually rely on a set of ambient sensors able to collect indoor values of 50 temperature and humidity. This allows the control system to 51 build thermal maps of the indoor environment, locate thermal 52 complaint feedbacks coming from the tenants and regulate ${ }_{53}$ only the necessary portion of the physical system. Another 54 target feature of complex buildings is safety, characterized by 55 the ability to respond to crisis events limiting damages and 56 victims. These systems are able to detect safety threats, for ${ }_{57}$ example from smoke detectors or heat detectors. Also in this scenario, a proper allocation of sensor nodes is essential to detect and locate the threat responsively.

The position of each node strongly affects the performance 61 of the system, since a bad allocation could lead to unmonitored 62 areas. The number of nodes employed, besides weighting on 63 the installation cost, also burdens the overall energy consump- 64 tion of the system, a key parameter to consider especially for 65 energy saving systems. The choice of the hardware nodes can 66 get more difficult by the availability on the market of several ${ }_{67}$ devices and components that differ in cost, power consumption 68 and maximum range distance. Although the key role of nodes allocation, many smart building systems proposed in literature do not consider nodes amount and positioning problems in environments that differ from the original testbeds.

Without a systematic approach the design space is not well 73 explored, which leads to inefficient solutions. In this con- 74 text, the development of tools able to automatize part of the 75 design flow of smart building systems is essential. In order 76 to find a near-optimal allocation of nodes, the knowledge of 77 the floor plan is required. However, for installations performed 78 on existing buildings, administrators can encounter difficulties 79 
TABLE I

\begin{tabular}{|l|c|c|c|c|}
\hline \multicolumn{1}{|c|}{ Deployment } & Site Specific vs General Model & Heterogeneous Nodes & Application Integrated & Extensible \\
\hline Zhao et al. [4] & General Model & No & Yes & No \\
\hline He at al. [5] & General Model & No & No & No \\
\hline Fang et. al. [6] & Site Specific Model & No & No & No \\
\hline Proposed approach & Site Specific Model & Yes & Yes & Yes \\
\hline
\end{tabular}

80 in obtaining the floor plan in an easily-interpretable digital 81 format.

82 To address these problems, we developed a computer-aided 83 design (CAD) tool to assist building designers during the 84 design of smart building systems. The application manages 85 common requirements like the building floor plan specifica86 tion. We decided to implement a node allocation algorithm 87 for three different indoor localization systems, that searches for 88 near-optimal allocations of nodes, from mixed hardware types, 89 with the aim of keeping low the total cost. Due to the high level 90 of heterogeneity and lack of standardization across systems to 91 design, we make the system extensible through plug-ins to let 92 new functionalities being integrated into the system. The tool ${ }^{1}$ ${ }_{93}$ is developed within the $\mathrm{QCAD}^{2}$ environment, an open-source 94 computer-aided drafting application. The key contributions of 95 this paper can be summarized as follows.

\section{RELATED WORK}

Building information modeling (BIM) is a consolidate 111 process to support building constructions and renovations. 112 BIM softwares, and in particular CAD for buildings such as ${ }_{113}$ ArchiCAD [7], focus on the generation and management of 114 digital representations of the physical aspects of places. BIM 115 tools can coordinate architectural and structural requirements, 116 for essential tasks such as collision detection [8]. Materials 117 employed for a construction can be represented with extremely 118 high levels of accuracy, thanks to the several libraries devel119 oped in many years, resulting in precise cost estimations [9]. 120 With the diffusion of integrated smart systems built to increase 121 comfort and efficiency, buildings require the design of aspects 122 that go beyond the mere physical design. The concept of smart

\footnotetext{
${ }^{1} \mathrm{~A}$ video demo of the tool has been published at https://youtu.be/6c6D6wolDBQ.

${ }^{2}$ QCAD—Open Source CAD System: http://www.qcad.org/.

${ }^{3}$ The source code of the system is open-source and available at https://bitbucket.org/necst/box-smartcad.
}

environment is becoming more and more concrete with the ${ }_{123}$ integration of sensors, actuators and computational elements 124 in buildings, while tools able to model smart and interactive ${ }_{125}$ functionalities of modern buildings are currently lacking. $\quad 126$

The problem of the allocation of hardware nodes in a given ${ }^{127}$ environment can be compared, on first approximation, by the ${ }_{128}$ maximal cover location problem (MCLP), i.e., the problem ${ }_{129}$ of covering the maximum amount of demand locations with 130 a given number of facilities. Similarly, the location set cov- ${ }_{131}$ ering problem (LSCP) consists in finding the minimum set ${ }_{132}$ of facilities that covers all available demand locations. Each ${ }_{133}$ facility has the same coverage radius $r$; a demand point is ${ }_{134}$ assumed to be covered if it is within distance $r$ of a facil- ${ }_{135}$ ity. Daskin et al. [10], [11] gave a general formulation of the 136 LSCP and reformulated it for network systems and emergency ${ }_{137}$ vehicle deployment.

The maximum sensing coverage region is a special case ${ }_{139}$ of the previous two problems that focuses on the research of 140 an allocation of wireless nodes that guarantees both sensing 141 coverage and network connectivity between nodes [12], [13]. 142 In this scenario, the placement need to take care not only of 143 the sensing range, but also of the communication range of each 144 node.

For what concern the allocation in indoor environments, 146 only minimum literature has been published so far to the ${ }_{147}$ best of our knowledge. Zhao et al. [4] proposed an AP posi- 148 tioning model based on the differential evolution algorithm, 149 specific for fingerprinting localization techniques. Their model 150 focuses on increasing the diversity of the received signal array 151 along the indoor locations, and thus improving the position- 152 ing accuracy of fingerprinting schemes. However, the model ${ }_{153}$ does not take into account the effect of walls or other obsta- 154 cles present in the target environment. He et al. [5] made use 155 of a genetic algorithm for APs deployment model, to study 156 the relationship between positioning error and signal space ${ }_{157}$ Euclidean distance. Again, the simulation results show that ${ }_{158}$ the error can be reduced increasing the Euclidean distance ${ }_{159}$ between the received signal strength (RSS) arrays of differ- 160 ent locations. Fang and Lin [6] proposed a tool for linking 161 the placement of APs and the positioning performance. Their 162 algorithm maximizes signal-to-noise ratio, i.e., maximizes the ${ }_{163}$ signal and minimizes the noise simultaneously. However, the ${ }_{164}$ system is developed in a real-world environment, and requires 165 measurements with different AP allocations that can be an 166 expensive and time-consuming task.

A common limitation of many works described previously is 168 the employment of simple and general models which does not ${ }_{169}$ take into account the actual layout and geometry of the build- 170 ing. The free-space path loss propagation model is often used 171 


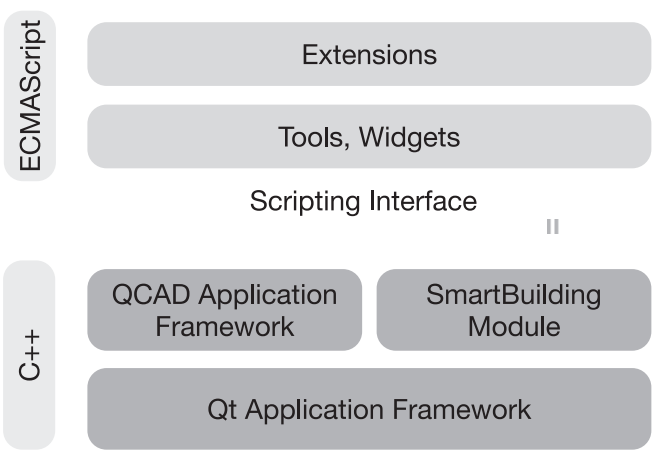

Fig. 1. Overview of the application stack. The script interpreter features standard ECMAScript functionality and on top of that provides additional classes from the Qt API, QCAD API, and the SmartBuilding module.

172 despite the presence of fixed obstructing objects like walls. Of 173 course, none of the cited works provide a convenient way to 174 specify geometric layout of the indoor environment. This leads 175 the authors to validate models simply using squared or rectan176 gular areas to represent the indoor environment, omitting the 177 relationship between irregular areas and system coverage. In 178 addition, none of the existing solutions takes in consideration 179 different hardware characteristics and costs of the nodes to be 180 deployed.

\section{Proposed Application Framework}

182 Our system has been developed on top of the QCAD appli183 cation framework. The QCAD application framework consists 184 of programming libraries and resources that provides CAD 185 specific functionalities. An example of module provided by 186 the QCAD application framework is the Math module that 187 implements mathematical concepts such as vectors or matri${ }_{188}$ ces as well as basic geometrical classes like points, lines and 189 so on. The QCAD Framework has been enhanced with a 190 SmartBuilding module that provides some fundamental func191 tionalities for the design of smart building systems. The 192 module include abstract entities like rooms, walls, sockets, 193 sensor nodes and gateways. User interface components are also 194 provided in order to create and edit this entities (tools) and to 195 specify parameters (widgets). Our module implements a node 196 deployment algorithm for three commons indoor localization 197 systems, that will be discussed later. The whole application 198 rely on Qt, a framework that covers a lot of generic and low199 level functionality for desktop applications and not directly 200 related to CAD.

201 The QCAD application framework offers a very complete 202 and powerful ECMAScript interface. The SmartBuilding mod203 ule, as well as the QCAD application framework, is accessible 204 through that scripting interface. Through the ECMAScript 205 interface developers will be able to extend the whole appli206 cation in an easy and very efficient way. The choice of a 207 popular script language that is easy to learn enables anyone 208 with previous programming experience to extend the appli209 cation. Such extensions can for example be CAD related 210 interactive tools like an HVAC layout construction widget, or 211 a temperature sensor nodes deployment algorithm.

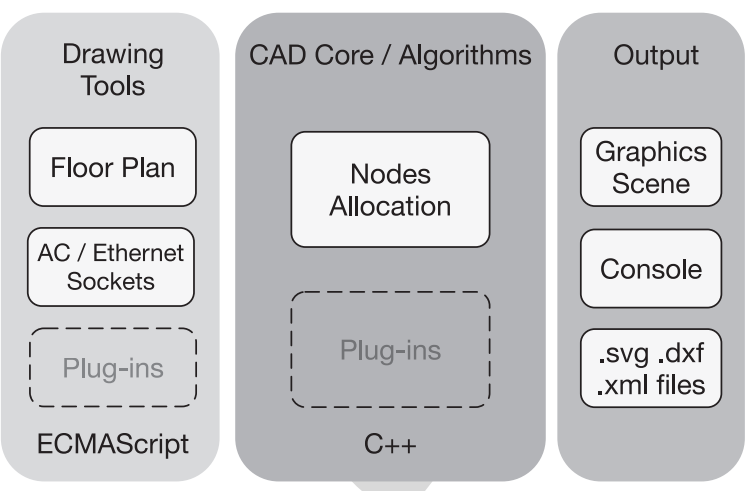

Fig. 2. Functional overview of the system components. Drawing tools and algorithms for systems deployment and simulation are extensible through ECMAScript or $\mathrm{C}++$ plug-ins.

In some situations extending QCAD through scripts alone 212 may not be possible. This is mostly the case, if the extension ${ }^{213}$ is based on an existing $\mathrm{C}$ or $\mathrm{C}++$ library. In that case, it is 214 possible to create a $\mathrm{C}++$ plug-in that wraps the existing library 215 and adds the necessary hooks to access library functionality 216 through the script interface. Such a plug-in will be automati- ${ }^{217}$ cally loaded by QCAD on start up to add functions and classes ${ }_{218}$ to the script interface of QCAD. These script extensions can ${ }_{219}$ then be used by a script add-ons to make that functionality 220 available as part of the application interface.

221

\section{NODES DePloyment FOR INDOOR LOCALIZATION}

Smart environments always rely on a set of hardware nodes 224 able to collect sensing data and communicate through cabled 225 or wireless technologies. The number of nodes employed and 226 the position of each one strongly affect the overall performance 227 of the system as well as the cost of installation. In this paper, 228 indoor localization systems have been taken as the main case 229 study for the nodes allocation, since occupants localization 230 and monitoring is one of the most common requirements of 231 different smart environments.

The way in which the indoor environment must be cov- 233 ered by the nodes depends on the particular technology 234 implemented; however, there can be identified three main ${ }_{235}$ manners.

1) Single coverage, i.e., to monitor the state of the envi- ${ }_{237}$ ronment with a single node for each location inside its 238 radius. This includes for example to detect the presence 239 of a mobile device in a proximity region [14], or to 240 detect an RFID tag within the tags reader range [15]. ${ }_{241}$

2) Trilateration, to compute the position of a mobile device. 242 This technique requires the reception of a wireless signal 243 of at least three reference sensors with well-known posi- 244 tions everywhere within the covered area. We define the 245 term $k$-coverage as the minimum number of sensors (or 246 reference nodes) required in each location by a system. ${ }_{247}$ Single coverage systems have $k$-coverage $=1$, while for 248 trilateration $k=3$.

3) Fingerprinting, where the number and the strength of the 250 received signals is not fixed, but affect the localization 251 accuracy.
252 


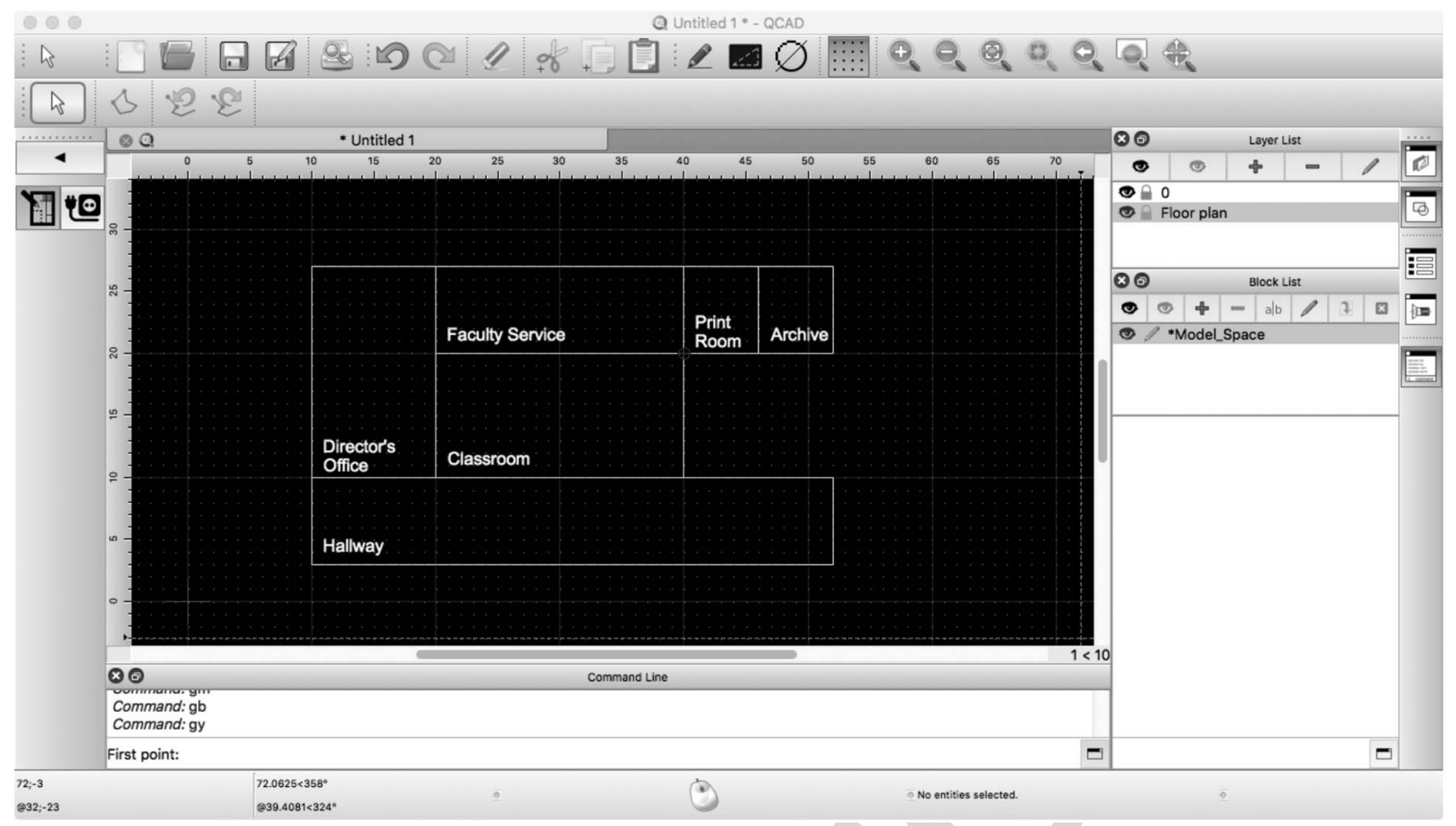

Fig. 3. Floor-plan design tool. User can specify the layout of the rooms and a possible set of candidate sites for the node placement.

253 Trilateration and fingerprinting usually exploit wireless tech254 nologies as Wi-Fi or Bluetooth to establish a connection 255 between mobile and stationary nodes. Sensing regions can 256 refer to any type of ambient sensors, such as passive infrared 257 sensors [16], remote thermal sensors [17], but also proximity258 based radio transmitters such as RFID tag readers [18] and 259 Bluetooth low energy transmitters (BLE beacons) [19].

\section{Proposed Deployment TOOL}

261 As we previously said, smart environments always rely on 262 a set of sensor nodes, each one able to communicate through 263 cabled or wireless technologies. Also for outdoor WSNs, a key 264 challenge is how to achieve coverage of the target monitor265 ing space and sufficient network connectivity between sensor 266 nodes. Usually each sensor mote communicates with the rest 267 of the network through technologies like Wi-Fi or ZigBee. 268 Additional issues for outdoor WSNs are the limited battery life 269 of each node and the power consumption required for packet 270 transmissions. Given the availability in most (also "nonsmart") 271 buildings of power outlets, Ethernet sockets and Wi-Fi sig272 nal, the mentioned limitations of WSNs can be solved in 273 indoor application making use of the existing infrastructure. 274 Differently from outdoor WSN deployments, where cover275 age and connectivity are always treated together, our system 276 leaves nodes connectivity optional, focusing on providing the 277 coverage service to the indoor locations.

278 The design process starts with a drafting phase in which the 279 user specify the building floor plan as a set of rooms. During 280 this phase the designer can restrict the possible sites for nodes 281 allocation, selecting a set of candidate points. This can be 282 useful when the hardware devices require power supply or
Ethernet connectivity. The design interface used for both map 283 and candidate sites specification is reported in Fig. 3.

In our model, we will refer to $L$ as the entire set of monitor- 285 ing locations to be covered, while $J$ as the set of deployable 286 locations where nodes can be placed. By default, $L=J$ and ${ }_{287}$ nodes can be positioned everywhere but as we said the set $J{ }_{288}$ can be restricted only to specific candidate points. 289

After the design phase, different parameters are provided by 290 the administrator and used to define a domain in which search 291 for a covering solution. The parameters are as follows. 292

1) The covering technique (single, trilateration, or fin- 293 gerprinting) that will be used to cover the locations 294 in $L$.

2) A cost $c_{t}$ for every type $t \in T$ of node available on the 296 market (expressed in dollars).

3) A working range $r_{t}$ for every type $t$ of node (expressed 298 in meters).

4) A percentage of covered area required, called target (i.e., 300 the minimum percentage of locations $l \in L$ to be covered 301 by the solution).

The system will return to the designer a set $N$ of nodes $n_{j t}{ }_{303}$ (possibly with mixed hardware types) and their position on 304 the building map. The outcome will have the lower cost of 305 installation among all the inspected solutions that satisfy the 306 target percentage of covered area. Fig. 4 shows an overview 307 of the process explained so far.

\section{A. Covering Techniques}

Our tool provides three different ways to cover the floor- 310 plan space, each one identified by the technique required by 311 the system that will be installed. 4 85 .

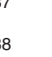
89 0

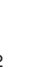
293
294 295 296 297 98 299 00 301

02

.

.

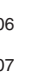
307
308 


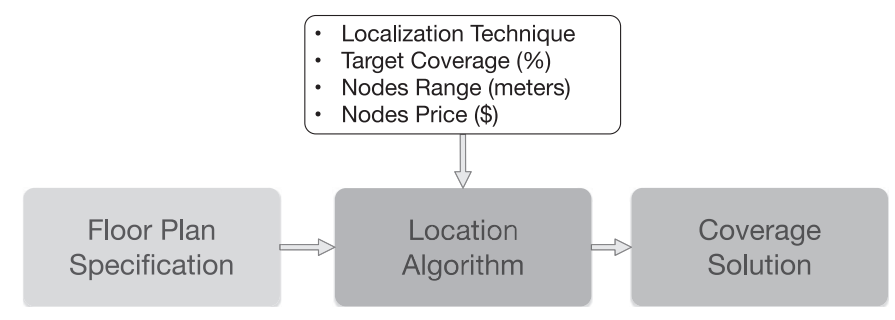

Fig. 4. System process. After the design of the floor plan, different parameters are used to define the search for an optimal allocation of nodes.

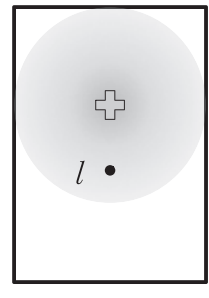

(a)

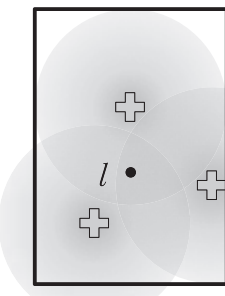

(b)

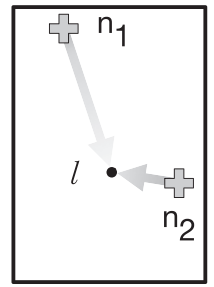

(c)

Fig. 5. Sample floor-plans with a location $l$ covered (a) in single mode, (b) for trilateration, and (c) for fingerprinting where $\operatorname{rss}_{l, 1}<\operatorname{rss}_{l, 2}$.

1) Single coverage that guarantees from each position the presence of at least one reachable node. This is used for example to detect the presence of a mobile device in a proximity region. In our model, a location $l$ of the floorplan is considered covered if exists at least one working node $n$ of type $t$ within a range $r_{t}$. An example is shown in Fig. 5(a).

2) Trilateration: This is the process of determining the position of a point measuring its distance from three reference nodes, exploiting geometric properties of triangles. Usually, indoor trilateration systems use the strength of the signal received from a node to estimate its distance. In our model, a location $l$ of the floor-plan is covered for trilateration if there exist at least three working nodes $n_{1}, n_{2}$, and $n_{3}$, each one no more distant then its corresponding range $r_{t}$. A location $l$ served for trilateration is shown in Fig. 5(b). Although we refer only to trilateration, the same exact result can be used also for triangulation, the technique where angles are measured instead of distances.

3) Fingerprinting: This technique is used to estimate the position of a mobile device based on its rss vector. Each location receives the signal from $k$ nodes, where $k$ is not the same for all locations, but depends on how many nodes are reachable from that particular location. Each one of the $k$ signals reaches the receiving antenna with a given power (or rss). For example, the location $l$ shown in Fig. 5(c) perceives $k=2$ signals so that $\operatorname{rss}_{l, 1}<\operatorname{rss}_{l, 2}$. We denote as $\operatorname{rss}_{l, n}$ the signal strength received at location $l$ from a node $n$. The vector $\operatorname{rss}_{\mathbf{I}}=\left[\mathrm{rss}_{l, 1}, \ldots, \operatorname{rss}_{l, k}\right]$ of the $k$ signals received at run-time in location $l$ is compared with a dataset of vectors, each one prelabeled with the corresponding position.
The comparison is usually performed by a classification 346 algorithm using the Euclidean distance of the vectors, since ${ }_{347}$ rss vectors with a small Euclidean distance between them are 348 more likely to be close also in the physical space. We have ${ }_{349}$ defined as $\operatorname{rss}_{l, n}$ the signal strength received at location $l$ from 350 a node $n$. The Euclidean distance between $\operatorname{rss}_{\mathbf{a}}$ and $\operatorname{rss}_{\mathbf{b}}$, both 351 composed by $k$ received signals, and collected, respectively, 352 in location $a$ and $b$ is defined as

$E(a, b)=\sqrt{\left(\operatorname{rss}_{a, 1}-\operatorname{rss}_{b, 1}\right)^{2}+\cdots+\left(\operatorname{rss}_{a, k}-\operatorname{rss}_{b, k}\right)^{2}}$.

Consider the vector $\mathrm{rss}_{\mathbf{a}}$ as the run-time sample, while 355 the vector $\mathrm{rss}_{\mathbf{b}}$ retrieved from the stored fingerprint. The 356 smaller is the $E(a, b)$, more confident is the localization system 357 approximating current location of $a$ with the stored location ${ }_{358}$ of $b$.

It has been demonstrated that maximizing the Euclidean 360 distances of the rss arrays between all sampling points, the 361 positioning accuracy of wireless localization systems can be 362 improved [4], [5]. Fig. 6 is reported a graphical demonstra- 363 tion of the aforementioned statement. Take as an example a 364 dataset (DS1, DS2, DS3, DS4) of stored rss vectors, where ${ }_{365}$ each vector is bi-dimensional $(K=2)$ and coupled with the 366 corresponding physical position. Fig. 6(a) shows each element ${ }_{367}$ of the database where the Cartesian coordinates corresponds to ${ }_{368}$ components $\mathrm{rss}_{1}, \mathrm{rss}_{2}$. Although the plane does not represent ${ }_{369}$ the physical area of the floor-plan, database elements that are 370 near between them are more likely to be close also in the phys- 371 ical space. Given a run-time element $R$, each arrow represents 372 the Euclidean distance $E\left(R, \mathrm{DS}_{i}\right)$ from the surrounding dataset ${ }_{373}$ elements. A localization algorithm can exploit the Nearest ${ }_{374}$ Neighbor technique to approximate the position of $R$ with 375 the nearest dataset element. Unfortunately, the run-time rss 376 measurement of $R$ will not be constant over time, but will 377 experience continuous fluctuations due to environmental noise. 378 These fluctuations make the sample $R$ move randomly to the 379 surrounding points. Suppose that DS2 is the nearest points to 380 $R$ in the physical space. Fig. 6(b) shows with a green area the ${ }_{381}$ probability to assign $R$ the correct (or more accurate) position, 382 while a red (with line pattern) area represents the probability ${ }_{383}$ to get a wrong position from the system. Fig. 6(c) demon- 384 strates how an increase in the rss Euclidean distance between 385 sampling points increase the red area and the accuracy of the ${ }_{386}$ localization, while in Fig. 6(d) an Euclidean distance reduction ${ }_{387}$ will lead to poorer localizations.

The RSS has been estimated using the The WINNER II ${ }_{389}$ path loss model [20]

$$
P L=A \log _{10}(d[m])+B+C \log _{10}\left(\frac{f_{c}[\mathrm{GHz}]}{5.0}\right)+X
$$

where $P L$ is the signal path loss (in $\mathrm{dB}$ ), $f_{C}$ is the frequency 392 in $\mathrm{GHz}$, and $d$ is the distance between the transmitter and ${ }_{393}$ the receiver location in meters. Values of coefficients $A, B, C, 394$ and $X$ change depending on line-of-sight (LOS) or nonline-of- 395 sight (NLOS) propagations, and are reported in Table II. The 396 propagation model has been used in fingerprinting coverage to 397 maximize the Euclidean distance of the rss vectors between a 398 location and its surrounding points, with the aim of improve 399 the localization accuracy of the system. 


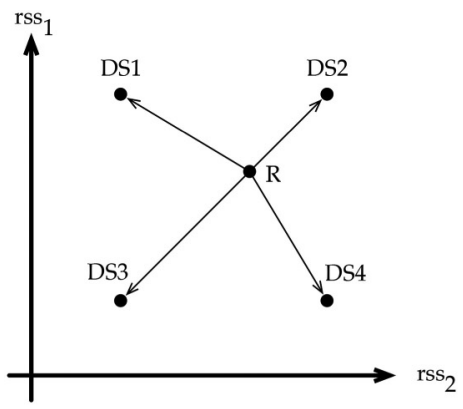

(a)

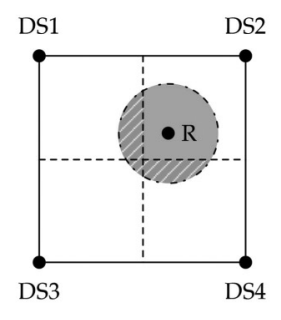

(b)

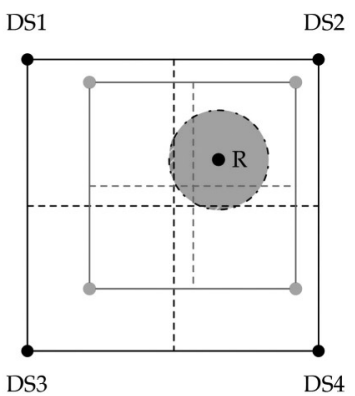

(c)

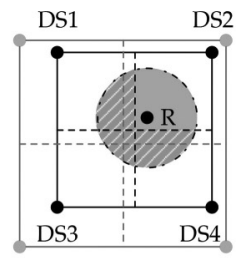

(d)

Fig. 6. (a) Bi-dimensional elements of the localization dataset are represented in Cartesian coordinates corresponding to components rss 1 and rss 2 . A run-time sample $R$ is shown in (b) where its circular area delineates run-time signal fluctuations. If DS2 is the nearest points to $R$ in the physical space, green area is proportional to the probability of correct localization, while red dashed area represent wrong localizations. (c) Euclidean distance between sampling points has been increased, improving the correct localization. (d) Opposite effect.

TABLE II

VALUES OF COEFFICIENTS DEPENDING ON LOS OR NLOS Propagations. Values HaVe BeEn TAKen From THE WINNER II PATH LOSS MODEL [20]

\begin{tabular}{|c|c|}
\hline Scenario & Path Loss Coefficients \\
\hline$L O S$ & $A=18.7, B=46.8, C=20$ \\
\hline$N L O S$ & $\begin{array}{l}A=36.8, B=43.8, C=20 \\
X=5\left(n_{w}-1\right) \text { (light walls) } \\
X=12\left(n_{w}-1\right) \text { (heavy walls) }\end{array}$ \\
\hline
\end{tabular}

401 The 2-D space of the floor plan is discretized with a length 402 unit (default is $1 \mathrm{~m}$ ) that is chosen by the user during the map 403 specification phase.

404 As we have said, in addition to location coverage, also nodes 405 connectivity has been modeled. In our model, a sensor node $n$ 406 is connected if exist a connected path to the gateway node. To 407 ensure the connectivity of the whole network, the following 408 equation must hold:

$409 \quad \forall n \in N$, connected $(n$, gateway) $=$ true

410 where

$$
\begin{aligned}
& 411 \\
& 412 \\
& 413 \\
& 414 \\
& 415
\end{aligned}
$$$$
416
$$
417 rithm in the same way of nonconnected networks, with the 418 following exception.

419 1) First, a manual gateway nodes allocation is required.

420 2) During nodes allocation, deployable points $421 \quad J$ are restricted to locations $j^{\prime}$ such that $422 \quad \operatorname{connected}\left(n_{j^{\prime}}\right.$, gateway $)=$ true.

423 3) During deployment optimization, nodes moves are considered feasible only within the connected area.

\section{Covering Location Algorithm}

\begin{tabular}{|c|c|}
\hline Notation & Meaning \\
\hline$L$ & set of monitoring locations \\
\hline$J$ & set of deployable locations \\
\hline$c_{t}$ & cost of a node of type $t$ \\
\hline$r_{t}$ & sensing range of a node of type $t$ \\
\hline$h_{t}$ & communication range of a node of type $t$ \\
\hline target & coverage rate of $L$ required by user $(\%)$ \\
\hline$n_{j t}$ & nodes of type $t$ allocated in $j$ \\
\hline$r s s_{l, n}$ & signal strength received in $l$ from $n$ \\
\hline $\mathbf{r s S}_{\mathbf{a}}$ & vector of all the $r s s_{a, n}$ values collected in $a$ \\
\hline$E(a, b)$ & Euclidean distance between $\mathbf{r s s}_{\mathbf{a}}$ and $\mathbf{r s s}_{\mathbf{b}}$ \\
\hline$D_{l}$ & set of locations no more distant than $d$ from $l$ \\
\hline$z$ & average signal space Euclidean distance \\
\hline$Z$ & objective function \\
\hline$b_{l}$ & reward earned for covering location $l$ \\
\hline$w_{l}$ & reward weighted on the node cost \\
\hline$x_{j t}$ & allocation of node with type $t$ in $j$ (binary) \\
\hline$a_{l j t}$ & reachability of $n_{j t}$ from location $l$ (binary) \\
\hline$k$-coverage & number of ref. nodes required by the system \\
\hline$k_{l}$ & current number of ref. nodes covering $l$ \\
\hline$S$ & min. signal space Euclidean distance threshold \\
\hline$s_{\min }$ & minimum number of node moves in shaking procedure \\
\hline$s_{\max }$ & maximum number of node moves in shaking procedure \\
\hline$R \max$ & number of restarts of the $V N S$ algorithm \\
\hline
\end{tabular}

426 The covering location algorithm has the purpose of plac427 ing an optimal set of nodes on the building floor plan.
TABLE III

Notation and Meaning of Symbols Used for the Model

We have decided to implement a modified version of the 428 multimode covering location problem [21], a generalization 429 of the MCLP. Using a quite general and flexible reformu- 430 lation of the covering problem, we have been able to adapt 431 the algorithm at the different covering techniques described ${ }_{432}$ previously.

The positioning algorithm is composed by a first Greedy 434 procedure, whose solution is then improved by a variable 435 neighborhood search (VNS) algorithm. The positioning algo- 436 rithm evaluates different solutions using a reward $b_{l}$, that is 437 defined for each location $l$ and will be earned only for the 438 locations covered in that particular solution. The value of the 439 reward depends on the coverage technique.

1) Single Coverage: The reward $b_{l}$ will be earned if there 441 is at least one node that covers $l$.

2) Trilateration: The reward $b_{l}$ will be earned if there are 443 at least three nodes that cover $l$. 440 41 42 . 443
444 5 (n) . (n) 


$$
\begin{gathered}
\mathbf{r s s}_{\mathbf{l}}=\left\langle r s s_{l, 1}, r s s_{l, 2}\right\rangle=\langle-84,-72\rangle[d B] \\
\mathbf{r s s}_{\mathbf{s}}=\left\langle r s s_{s, 1}, r s s_{s, 2}\right\rangle=\langle-67,-41\rangle[d B] \\
E(l, s)= \\
=\sqrt{\left(r s s_{l, 1}-r s s_{s, 1}\right)^{2}+\left(r s s_{l, 2}-r s s_{s, 2}\right)^{2}}= \\
=\sqrt{(-84-(-67))^{2}+(-72-(-41))^{2}}= \\
=35.36[d B]
\end{gathered}
$$

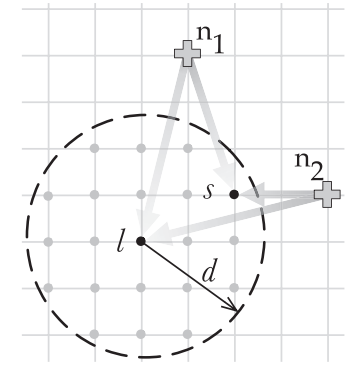

Fig. 7. Regular grid showing how is computed the mean Euclidean distance between the received rss vectors in a certain location $l$, and the surrounding locations $s$ within a certain distance $d$.

451 racy of the system it is possible to increase the signal space ${ }_{452}$ Euclidean distance between the target points. Consider the 453 mean Euclidean distance between the received rss vector in 454 a certain location $l$, and the surrounding locations $s$ within a 455 certain distance $d$

456

457

$$
\begin{aligned}
& \frac{1}{\left|D_{l}\right|} \sum_{s \in D_{l}} E(l, s) \\
& D_{l}=\{s \in L \mid \operatorname{distance}(l, s) \leq d\} .
\end{aligned}
$$

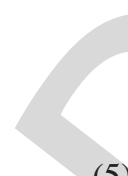

${ }_{458}$ The distance $d$ is used to restrict the rss comparison and 459 diversification only to the locations that are more likely to be ${ }_{460}$ erroneously confuse with $l$ by the localization system. Fig. 7 461 shows an example of how the Euclidean distance of a location 462 is compared to a neighbor location.

${ }_{463}$ We define the average signal space Euclidean distance $z$

464

$$
z=\frac{\sum_{l \in L} \sum_{s \in D_{l}} \frac{E(l, s)}{\left|D_{l}\right|}}{|L|} .
$$

465 The term $z$ will be used by the Greedy procedure to produce 466 a first solution with a reasonable allocation of nodes. Then, the 467 value of $z$ should be increased as much as possible to provide 468 good localization accuracy to the system. However, maximize 469 only the average does not seems fair enough, since a good 470 system should provide a certain level of accuracy homoge471 neously among the target area. So we defined the objective 472 function as difference between the term $z$ and the signal space 473 Euclidean variance

$$
Z=z-\sqrt{\sum_{l \in L}\left(\sum_{s \in D_{l}} \frac{E(l, s)}{\left|D_{l}\right|}\right)^{2}} .
$$

475 Maximizing the objective function $Z$, the intention is to 476 provide as many target location as possible with a high sig477 nal space Euclidean distance with respect to the surrounding 478 locations.
As we have previously introduced, we represent with $L$ the 479 entire set of location to be covered, while with $J$ the set of pos- 480 sible positions where nodes can be placed. By default, $L=J{ }_{481}$ and nodes can be positioned everywhere; however, its possi- ${ }_{482}$ ble to restrict the $J$ set only to specific candidate points, that ${ }^{483}$ represent for example power outlets or Ethernet sockets. The ${ }_{484}$ problem of find a near-optimal set $N$ of nodes $n_{j t}$ (each one ${ }_{485}$ located in $j$ and having a type $t$ ) with a coverage rate $f(N)$ that ${ }_{486}$ satisfies the target coverage, can be formalized as follows: ${ }_{487}$

$$
\begin{aligned}
& \max Z=z-\sqrt{\sum_{l \in L}\left(\sum_{s \in D_{l}} \frac{E(l, s)}{\left|D_{l}\right|}\right)^{2}} \\
& f(N) \geq \operatorname{target} \\
& \sum_{t \in T} x_{j t} \leq 1 \quad \forall j \in J \\
& x_{j t}=1 \quad \Longleftrightarrow n_{j t} \in N \\
& f(N)=|L| / \sum_{l \in L} y_{l} \\
& \left\{\begin{array}{l}
y_{l} \leq \sum_{j \in J} \sum_{t \in T} a_{l j t} x_{j t} \quad \forall l \in L \text { (single) } \\
2 y_{l} \leq \sum_{j \in J} \sum_{t \in T} a_{l j t} x_{j t} \quad \forall l \in L \text { (fingerprinting) } \\
3 y_{l} \leq \sum_{j \in J} \sum_{t \in T} a_{l j t} x_{j t} \quad \forall l \in L \quad \text { (trilateration). }
\end{array}\right.
\end{aligned}
$$

The decision variable $x_{j t}=1$ represents the allocation of ${ }_{494}$ a node of type $t$ in location $j ; a_{l j t}$ is equal to 1 if location $l{ }_{495}$ can be reached by a node of type $t$ placed in $j$, and $a_{l j t}=0496$ otherwise. $y_{l}=1$ if location $l$ is covered, $y_{l}=0$ otherwise. 497 The constraint (10) fixes to one the maximum number of nodes 498 that can be located in each site.

\section{A. Greedy Procedure}

500

The positioning algorithm starts with a Greedy procedure 501 with the purpose of find a reasonable number of reference ${ }_{502}$ nodes, for both coverage and localization accuracy. The pro- 503 cedure generate a first solution $N$ positioning a set of $k=|N|{ }_{504}$ nodes, each one with a type $t \in T$. For all three coverage tech- 505 niques, the reward $b_{l}$ is weighted with the cost of the current 506 node $n^{*}$ selected for the coverage

$$
w_{l}=\frac{b_{l}}{c_{t}} ; \quad\left\{n^{*}=n_{j t} \wedge \operatorname{distance}(j, l) \leq r_{t}\right\} .
$$

The weighted reward $w_{l}$ will be used by the Greedy algorithm 509 so that on equal covered area, the cheapest node type has 510 the priority over the others. We denote as $L_{j t}$ the subset of 511 locations that are reachable by a reference node $n$ of type $t{ }_{512}$ placed at location $j$. At each iteration, the algorithm places 513 a node $n$ of type $t^{*}$ at position $j^{*}$ that covers the subset of ${ }_{514}$ locations $L_{j^{*}} t^{*}$ with the maximum reward. The term

515

$$
1-\frac{k_{l}}{k-\text { coverage }}
$$

is used to prioritize the covering of locations with a 517 lower "temporary" $k$-coverage (called $k_{l}$ ) with respect to the 518 $k$-coverage required by the current techniques. In this way, 519 Greedy procedure tends to avoid the placement of nodes very 520 


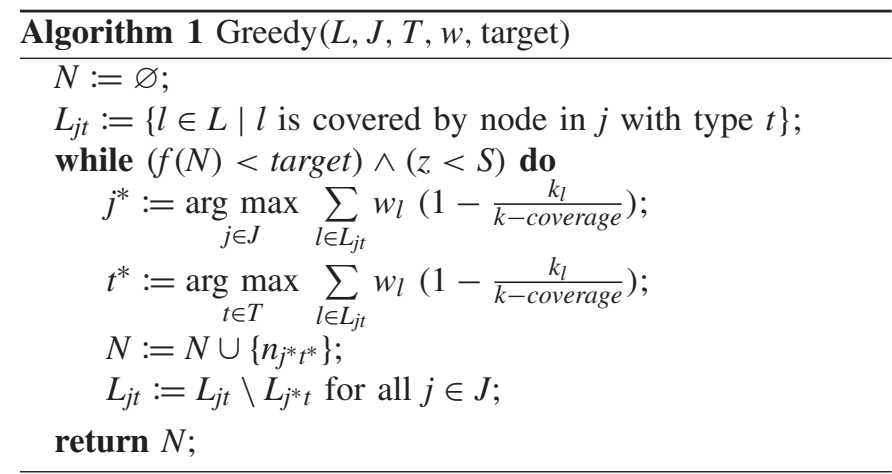

521 close to one other which can lead, especially for trilateration 522 systems, to poor localization accuracy. It is important to notice 523 that the purpose of the Greedy procedure is to find a reasonable 524 number of nodes for the localization service. The starting posi525 tioning is made on a best-effort basis, that will be improved 526 by the successive VNS. After a node allocation, all subsets ${ }_{527} L_{j t}$ are updated according to the coverage technique. In trilat528 eration for example, a location $l$ is removed from $L_{j t}$ only if 529 there exist, other than the current $n_{j^{*}} t^{*}$, other two nodes that 530 are already covering $l$.

${ }_{531}$ The Greedy procedure ends when the target coverage is sat532 isfied, and when the average signal space Euclidean distance 5 5з $z$ reaches the threshold $S$. In our implementation we set the 534 threshold $S=4.5$ that has been proven to be the average 535 Euclidean distance for which the positioning error is limited 536 to $2 \mathrm{~m}$ [5]. How we will see in Section VII, the Greedy proce537 dure is able to provide an average Euclidean distance not so far 538 from the final best known. However, thanks to the low com539 plexity of the Greedy procedure, additional time can be used 540 to improve the solution. In addition, the Euclidean distance 541 variance will be strongly improved.

\section{B. Variable Neighborhood Search}

${ }_{543}$ The method called VNS has been used to improve the solu544 tion coming from the Greedy procedure. The VNS approach 545 empowers the classical local search framework with a restart 546 mechanism that extends the search after a local optimum 547 has been achieved by generating new starting solutions in 548 progressively enlarged neighborhoods of the current best 549 known solution. The key elements of the VNS (reported in 550 Algorithm 2) are a starting solution $N$ with a hierarchy of 551 size-increasing neighborhoods, and a local search procedure, 552 i.e., the criterion to select the incumbent solution from the 553 neighborhood. These components are used to restart the search ${ }_{554}$ every time that the procedure reaches a local optimum. Fig. 8 555 shows an overview of the VNS process. A first local search 556 procedure is applied to the solution produced by the Greedy 557 procedure. At each iteration, the shaking procedure is used 558 to generate a new starting solution, which is then improved 559 by the execution of the local search. The shaking procedure 560 perturbs $s$ node allocations of the current solution $N^{*}$ replac561 ing them with $s$ unused nodes. The behavior of the shaking 562 parameter $s$, that depends on the result of the local search, is 563 explained in Fig. 9. The parameter $s$ starts from a minimum

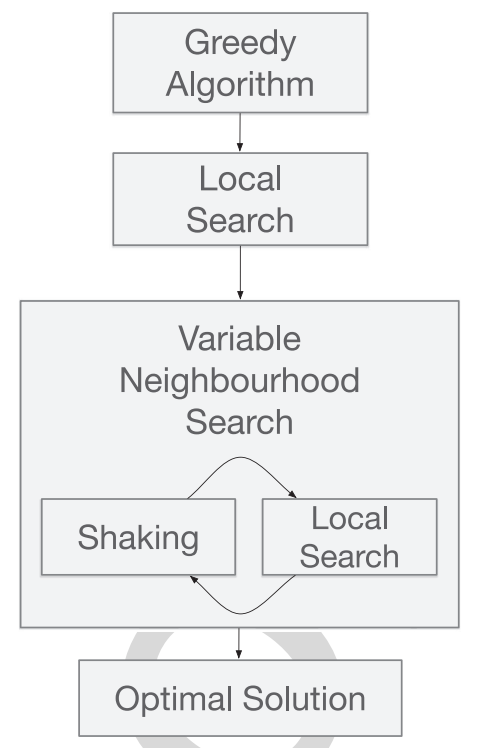

Fig. 8. Location algorithm. The solution found by the Greedy algorithm is improved applying iteratively a Local Search for an optimal solution and a Shaking procedure that perturbs the current solution.

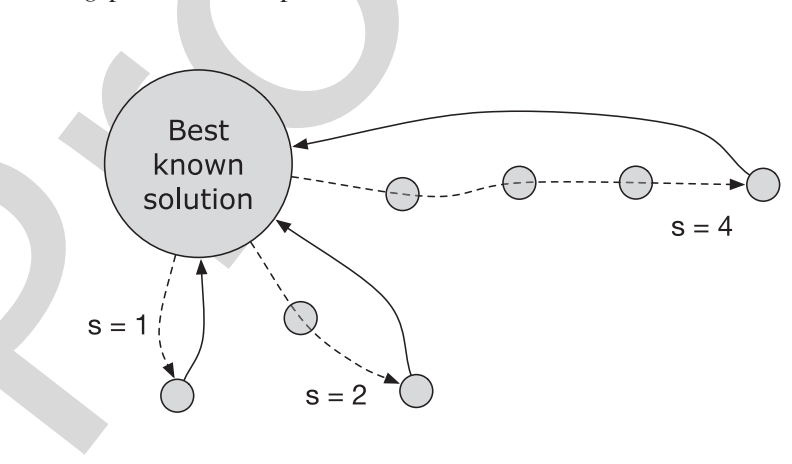

Intensification

Diversification

Fig. 9. Shaking procedure: the parameter $s$ is increased when the solution does not improve (dashed line) and restarts when a new optimum is found (continuous line).

value $s_{\min }$ (in the example $s_{\min }=1$ ) and every time that the 564 local search does not improve the best known solution, $s$ is 565 increased by 1 . Differently, when the local search succeeds, 566 the best solution $N^{*}$ is updated and $s$ goes back to $s_{\min }$. $\quad 567$

The purpose of the shaking procedure is to first explore 568 new starting solutions that are more similar to the best known 569 result, so that the search is intensified in a promising neigh- 570 borhood of the entire domain. If these local searches fail, the 571 shaking procedure moves the search from intensification to 572 diversification, generating starting solutions that are more and 573 more different from the incumbent one. Whenever a new best 574 solution is found, the shaking procedure comes back to $s_{\min }$, to 575 intensify the search near the just updated $N^{*}$. In principle, the 576 shaking parameter $s$ can be increased until $k=\left|N^{*}\right|$, changing 577 all the node allocations. However, we experimented running 578 different configurations that excessively moving away from 579 the best known solution can be unproductive, causing a use- 580 less waste of computational time. We have fixed a reasonable 581 value of $s_{\max }=\lfloor(2 / 3) k\rfloor$. 


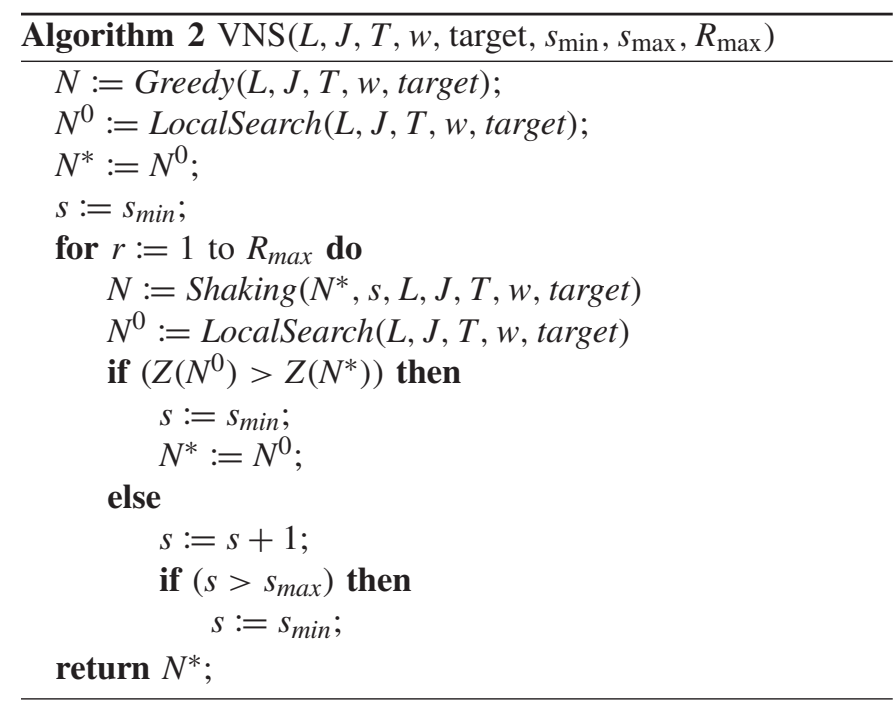

583 The VNS algorithm terminates when the total number of 584 restarts reaches a given value $R_{\max }$.

585 As we have said, the local search is the heuristic that 586 proceeds from an initial solution to its neighborhood by a 587 sequence of local changes, trying to improve each time the 588 value of the objective function until a local optimum is found. 589 The neighborhood of the adopted approach is given by cyclic 590 sequences of moves, where each move consists in locating a 591 new node, removing a node or changing the type of the node. 592 A cyclic move is considered feasible only if the new covering ${ }_{593}$ rate respects the target coverage, and the total cost of the solu594 tion does not increase. Of course, each site must continue to 595 hosts at maximum one node [constraint (10)]. A cyclic move 596 can be visualized on a graph $G=(N, A)$, where each node of 597 the graph is a possible allocation of a hardware node. Each 598 node of the graph is characterized by a location $j$, and a state 599 that indicates if the node is active or inactive. A node $n_{j t}$ cur600 rently allocated in location $j$, is represented on the graph with 601 an active node $n_{j}$, labeled with its hardware type $t$. Note that 602 index $t$ does not appear because at most one type can be active 603 in each node, and the type is specified by the label. Inactive 604 nodes are instead left unlabeled. An arc $\left(n_{j}, n_{k}\right)$ can represent 605 the following.

606 1) The allocation of a hardware node in site $j$, if $n_{j}$ is inactive and $n_{k}$ is active.

2) The removal of a hardware node in site $j$, if $n_{j}$ is active and $n_{k}$ is inactive.

610

611

3) An hardware node $n_{j}$ changing its hardware type, if both nodes are active.

612 In both 1) and 2), the new node takes the hardware type of 613 the head label ( $t$ of $n_{k}$ ). A cyclic exchange corresponds to 614 a directed cycle on the improvement graph, as depicted in 615 Fig. 10. Each move, and so each $\operatorname{arc}\left(n_{j}, n_{k}\right)$, determines a vari616 ation $\delta Z$ in the value of the objective function $Z$. The purpose 617 is to represent a group of moves so that a cyclic exchange rep618 resents an increase in the current objective function. However, 619 the total variation $\delta Z$ is non additive with respect to the 620 sequence of $\delta Z$ values coming from single moves. This is ${ }_{621}$ caused by the interdependence between different hardware

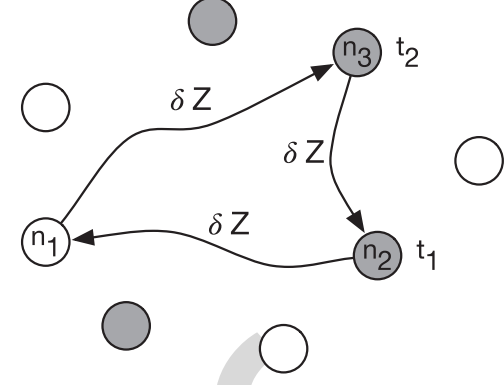

Fig. 10. Improvement graph: colored nodes represent current allocations, while empty nodes are possible allocations. All active nodes are labeled with their corresponding type. Each arc is a change (move) on the allocations.

nodes with overlapping covering regions, that lead to nonaddi- 622 tive moves. To overcome this drawback, every cycle has been ${ }^{623}$ evaluated using an own temporary function $Z^{\prime}$ updated step by ${ }_{624}$ step from the end of the path to its starting node. In this way, ${ }_{625}$ all the cycles with a positive total weight bring improvements 626 on the starting solution.

The search for the cyclic exchange with maximum weight ${ }_{628}$ is performed with exhaustive breadth-first exploration of the 629 paths of graph $G$.

\section{EXPERIMENTAL RESULTS}

631

Presented experimental results are initially focused on the 632 usability of the tool, testing the ability to provide a solution ${ }_{633}$ in a reasonable time. Then, the performances of the model ${ }_{634}$ have been evaluated, in terms of localization accuracy through ${ }_{635}$ realistic indoor environment experiments, and in terms of cost- ${ }_{336}$ effectiveness of the suggested deployments.

\section{A. Computational Experience}

638

The tool has been evaluated running several different config- 639 urations. Every test reported in this section has been executed 640 with a spatial resolution of the floor plan equal to $1 \mathrm{~m}$. A first ${ }_{641}$ analysis can be done on the execution times of the proposed ${ }_{642}$ solution. Although the execution time can be tuned by the ${ }_{643}$ parameter $R_{\max }$, which represents the maximum number of 644 restarts of the VNS algorithm, an idea on the order of mag- 645 nitude is given by Fig. 11, where the time is represented as 646 a function of the floor-plan dimension. In the given example, ${ }_{647}$ $R_{\max }$ has been fixed to 20 restarts, the target coverage equals 648 to $95 \%$ of the total area, a single node type available with a 649 range of $8 \mathrm{~m}$, covering floor-plans with rectangular areas. The 650 graph shows that for single coverage and fingerprinting the 651 processing time grows approximately linearly with the floor 652 plan area.

653

A numeric comparison of the same tests is reported in 654 Table IV, where execution times are reported in seconds for 655 increasing floor plans. For single coverage, the execution time 656 is low even for areas of 3000 squared meters. For trilatera- 657 tion and fingerprinting, the execution times become high from 658 floor-plan of $2500 \mathrm{~m}^{2}$. However, the tests represent a bad case 659 in which the map dimension is very large while the node range 660 available and the spatial resolution are small (respectively, 661 


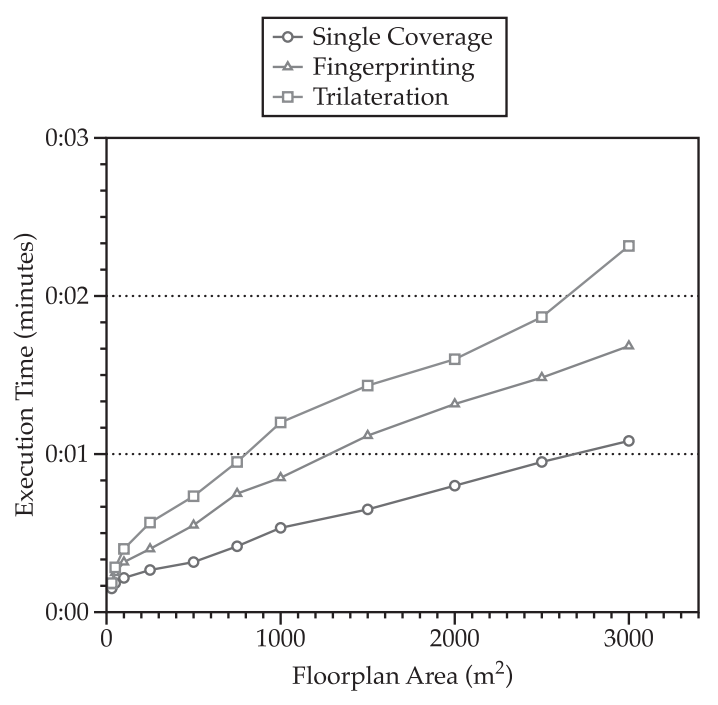

Fig. 11. Execution time of the tool with floor plans of different areas, for each covering technique $\left(R_{\max }=20\right.$, target $=95 \%$, and $\left.r_{t}=8\right)$.

TABLE IV

EXECUTION TIME OF THE TOOL FOR INCREASING FLOOR Plan AREAS $\left(R_{\max }=20\right.$, target $=95 \%$, AND $\left.r_{t}=8\right)$

\begin{tabular}{|c|c|c|c|}
\hline \multirow{2}{*}{$\begin{array}{c}\text { Floor Plan } \\
\text { Area }\left(\mathrm{m}^{2}\right)\end{array}$} & \multicolumn{3}{|c|}{ Execution Time (s) } \\
\cline { 2 - 4 } & Single & Fingerprinting & Trilateration \\
\hline 30 & 9.07 & 10.53 & 11.49 \\
50 & 11.31 & 15.10 & 17.67 \\
100 & 13.05 & 19.41 & 24.22 \\
250 & 16.57 & 24.89 & 34.16 \\
500 & 19.18 & 33.48 & 44.66 \\
750 & 25.43 & 45.32 & 57.94 \\
1000 & 32.19 & 51.68 & 72.83 \\
1500 & 39.37 & 67.12 & 86.27 \\
2000 & 48.30 & 79.49 & 96.11 \\
2500 & 57.11 & 89.47 & 112.34 \\
3000 & 65.41 & 101.24 & 139.18 \\
\hline
\end{tabular}

6628 and $1 \mathrm{~m}$ ). Increasing the range or the resolution, the instance ${ }_{663}$ of the problem decrease, resulting in faster executions.

${ }_{664}$ A key aspect that characterizes the goodness of the 665 proposed approach is the improvement of the objective func666 tion achieved by the VNS algorithm with respect to the first ${ }_{667}$ Greedy configuration. For this test we have run the tool sev668 eral times with a floor-plan area of $2500 \mathrm{~m}^{2}$ and a node range 669 of $12 \mathrm{~m}$. The number of reference nodes allocated is deter670 mined by the Greedy procedure and increase with $S$, while 671 the number of VNS restarts $R_{\max }$ has been fixed to 35 .

672 In Fig. 12, we reported the value of $z$, i.e., the average signal 673 space Euclidean distance obtained with the first Greedy exe674 cution, compared with the $z$ value after the VNS optimization. 675 The graph reports the $z$ values as a function of the threshold $S$, 676 described in Section VI-A as the minimum value of average 677 signal space Euclidean distance $(z)$ required during the Greedy 678 procedure. The graph shows that moving the threshold within

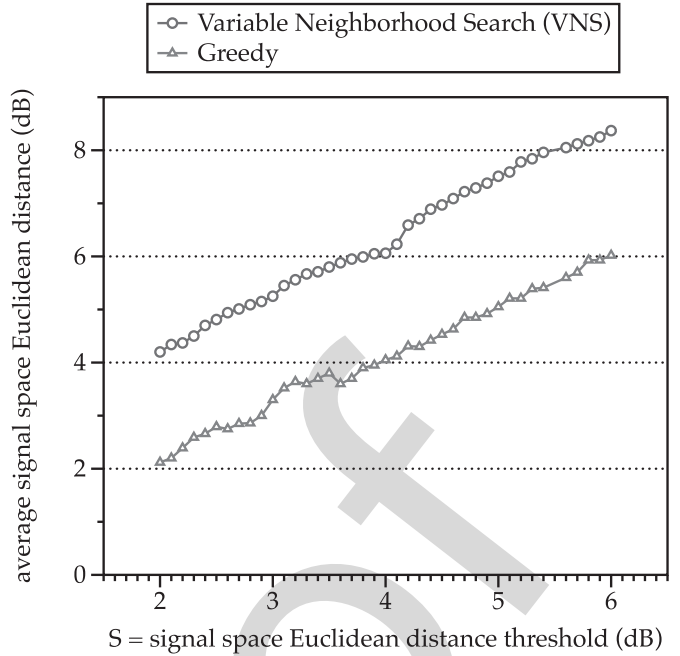

Fig. 12. Average signal space Euclidean distance $(z)$ obtained with the Greedy execution and compared with the $z$ value after the VNS optimization. $z$ values expressed as a function of the threshold $S$. Floor-plan area $=2500 \mathrm{~m}^{2}$, $R_{\max }=20$, target $=100 \%$, and $r_{t}=12$.
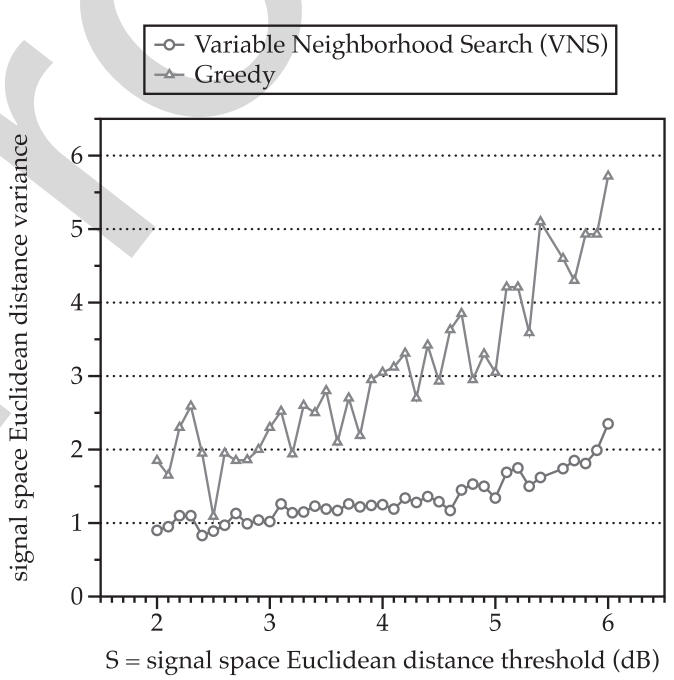

Fig. 13. Signal space Euclidean distance variance obtained with the Greedy execution and compared with the $z$ value after the VNS optimization. Values expressed as a function of the threshold $S$. Floor-plan area $=2500 \mathrm{~m}^{2}, R_{\max }=$ 20 , target $=100 \%$, and $r_{t}=12$.

the range $(2,6) \mathrm{dB}$ the VNS is able to improve the $z$ value con- 679 stantly around $2 \mathrm{~dB}$. Although the VNS improvement is not 680 astonishing for what regard the average value, Fig. 13 shows 681 that the variance is strongly improved. This has been achieved 682 moving from the objective function $z$ used in Greedy proce- ${ }_{683}$ dure to the $Z$ function of the VNS. The $Z$ objective function 684 has in fact the purpose to provide as many target location as 685 possible with a high signal space Euclidean distance w.r.t. the ${ }_{686}$ surrounding locations.

\section{B. Experimental Setup and Accuracy Evaluation}

688

The proposed tool was evaluated using data collected from 689 a real-world environment, the NECST Lab, located at the 690 basement of DEIB Department at the Politecnico di Milano. 691 


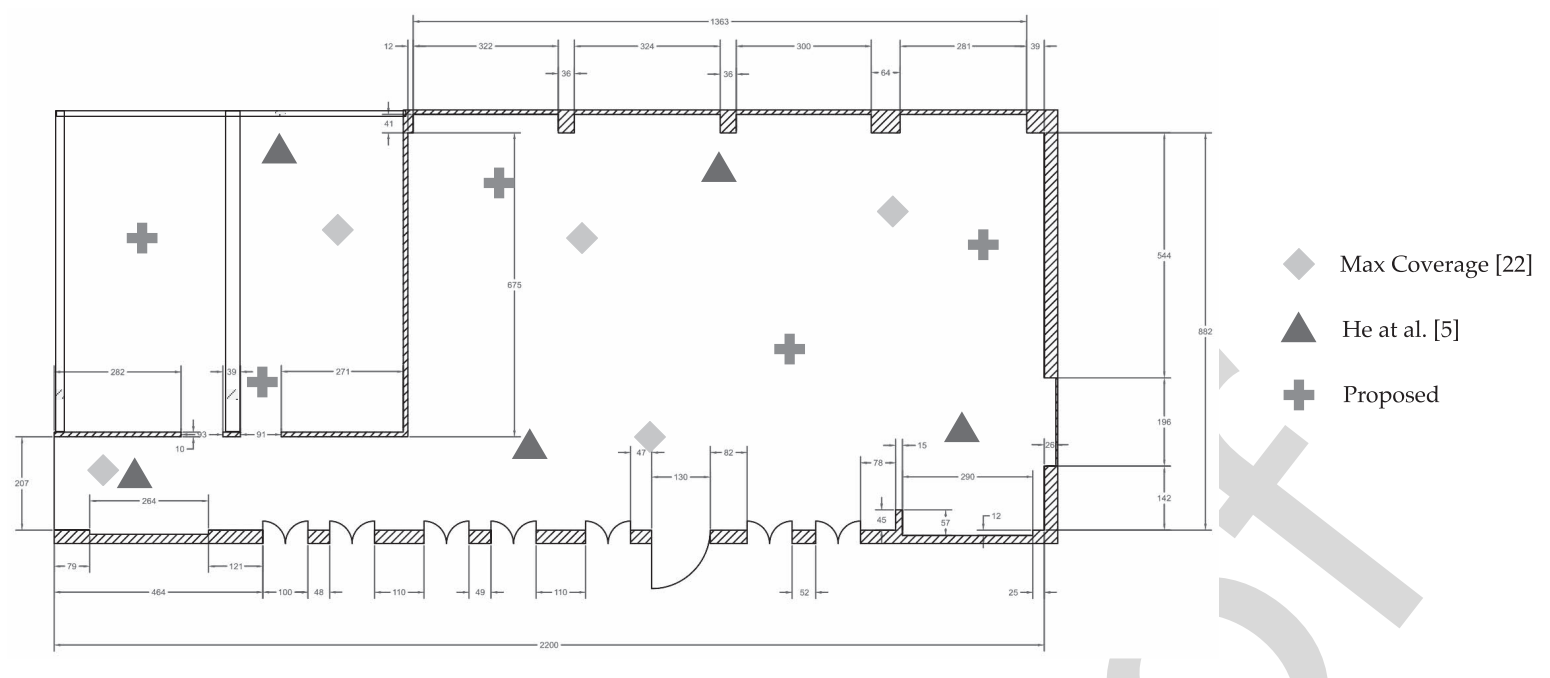

Fig. 14. NECST Laboratory floor-plan, located at the basement of DEIB Department at the Politecnico di Milano. Each allocation corresponds to a BLE beacon with a range of $7 \mathrm{~m}$. Green crosses indicates allocations provided by our algorithm, gray rhombus represent allocations from [5] while blue triangle positions have been computed maximizing the coverage [22].

692 The dimension of the test-bed is 198 squared meters $(9 \times 22 \mathrm{~m})$. ${ }_{693}$ We collected BLE signal data coming from BLE beacons with 694 a coverage radius of $7 \mathrm{~m}$. Signal data has been collected 695 using a Nexus 5 smartphone running Android 6.0.1. First, the 696 NECST Laboratory floor-plan has been designed using our 697 tool, obtaining the optimal number of beacons $(|N|=5)$ and 698 their allocation for fingerprinting localization. $R_{\max }$ has been 699 fixed to 20 restarts, the target coverage equals to $100 \%$ of the 700 total area, a single node type available with a range of $7 \mathrm{~m}$, and 701 the threshold $S=4,5$. We collected 40 training samples for 702 the localization algorithm using the obtained allocation. Then, 703 the test samples were collected at distinct positions changing 704 the phone orientation and the way in which user was keeping 705 it, for example by hand or in a pocket. For the entire duration 706 of training and test phase, the number of occupants and their 707 enabled wireless devices has changed, from a minimum of 3 to 708 a maximum of 17 people. This variation affects the accuracy 709 performances, but at the same time contributes in obtaining 710 realistic results. The training and test phase has been repeated 711 with two configurations coming from different allocation algo712 rithms: maximization of the coverage [22] and the allocation 713 algorithm proposed by He et al. [5]. For these two algorithms, 714 the number of employed nodes has been fixed to 5. KNN with $715 K=3$ has been employed as the fingerprinting algorithm.

${ }_{716}$ A first result is shown in Fig. 15. The cumulative error 717 distribution function shows that from $1.5 \mathrm{~m}$ our approach per718 forms better. Under $1.5 \mathrm{~m}$, He et al. [5] approach performs 719 better, but the difference in accuracy is marginal.

720 Fig. 16 shows the mean positioning accuracy divided into 721 different error ranges: $(0,0.5],(0.5,1],(1,1.5],(1.5,2]$, $722(2,2.5],(2.5,3],(3,3.5]$, and $(3.5,4]$. It is possible to notice 723 that the majority of the localization errors appears within the $724(1.5,2] \mathrm{m}$. The test-bed floor-plan, composed by three rooms, 725 has been reported in Fig. 14. Green crosses indicates allo726 cations provided by our algorithm, gray rhombus represent 727 allocations from [5] while blue triangle positions have been 728 computed maximizing the coverage [22].

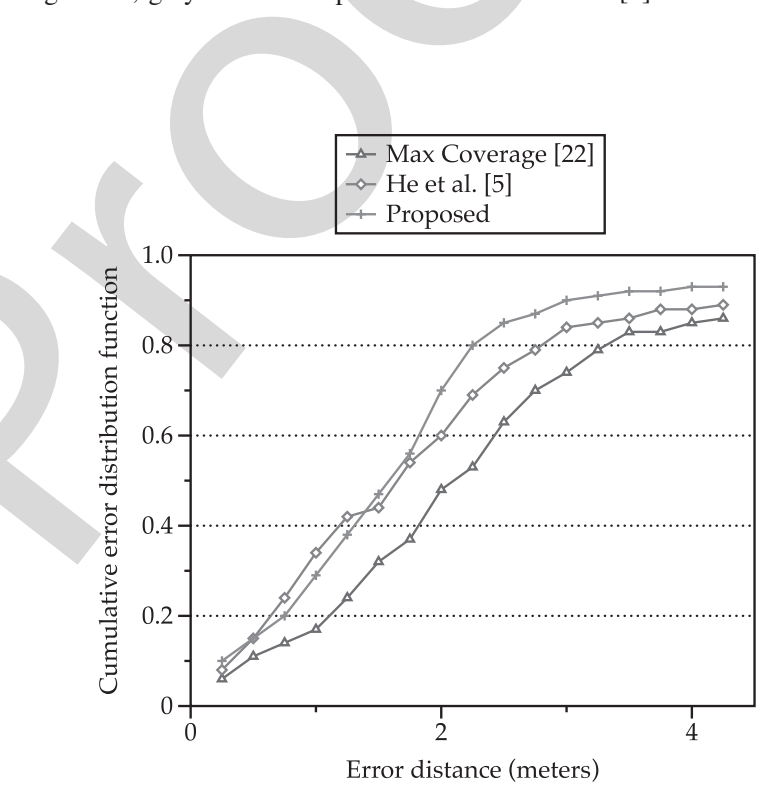

Fig. 15. Cumulative error distribution function experienced by our approach ad compared with two different solutions from the state-of-the-art.

\section{Cost-Effectiveness Analysis}

A feature of our tool interesting for testing is the possibility 730 to obtain solutions from mixed node types, with different char- 731 acteristics and costs. In particular, given two types $t_{1}$ and $t_{2}{ }_{732}$ characterized by two ranges $r_{i}$, and two costs $c_{i}$, it is possible ${ }_{733}$ to compare the total cost of a homogeneous solution with the 734 cost of a mixed solution. Given a baseline type of node with 735 a range $r_{1}=8 \mathrm{~m}$ and a cost of $c_{1}=60 \$$, we can assume ${ }_{736}$ the presence on the market of a second type of hardware, with 737 the half of the range distance $\left(r_{2}=4 \mathrm{~m}\right)$. The area covered ${ }_{738}$ by $t_{1}\left(\approx 200 \mathrm{~m}^{2}\right)$ is four times bigger than the coverage of $t_{2}{ }_{739}$ $\left(\approx 50 \mathrm{~m}^{2}\right)$. In order to obtain a fair test, the cost of $t_{2}$ should 740 be $c_{2} \geq c_{1} / 4$, and so we set $c_{2}=20 \$$. This test has been 741 performed with a target coverage of $95 \%$ on a rectangular map 742 of $1000 \mathrm{~m}^{2}$.

From Table V, it is possible to observe that, although hard- 744 ware nodes of type $t_{2}$ have a lower convenience in terms of 745 


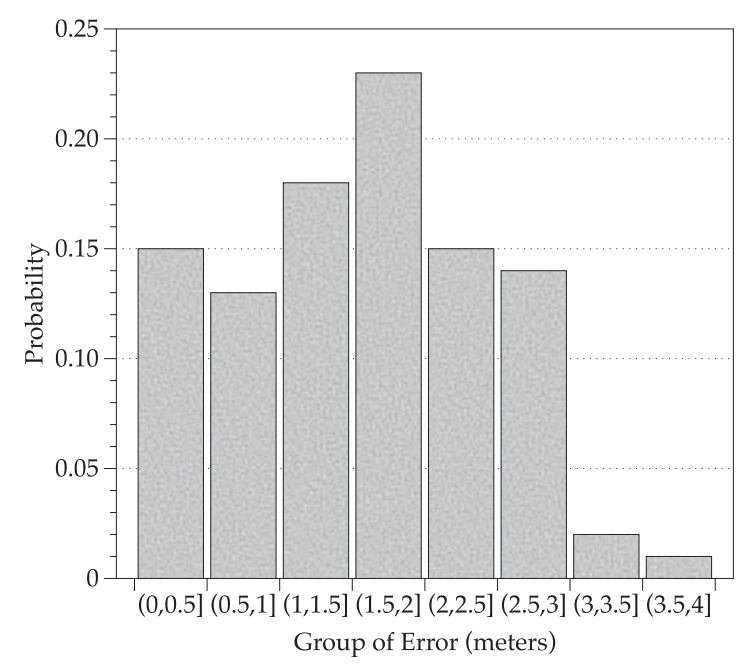

Fig. 16. Mean positioning accuracy of the proposed allocation algorithm divided into different error ranges.

TABLE V

Cost of Homogeneous and Mixed Solutions $\left(A=1000 \mathrm{~m}^{2}\right.$, target $=95 \%, r_{1}=8 \mathrm{~m}, r_{2}=4 \mathrm{~m}, c_{1}=60 \$$, AND $c_{2}=20 \$$ )

\begin{tabular}{|c|c|c|c|}
\hline \multirow{2}{*}{ Node types } & \multicolumn{3}{|c|}{ Solution Costs (in \$) } \\
\cline { 2 - 4 } & Single & Trilateration & Fingerprinting \\
\hline$T=\left\{t_{1}\right\}$ & 480 & 1440 & 840 \\
$T=\left\{t_{2}\right\}$ & 500 & 1620 & 880 \\
$T=\left\{t_{1}, t_{2}\right\}$ & 440 & 1280 & 760 \\
\hline
\end{tabular}

746 (area/price) ( $t_{1}$ outperform $t_{2}$ in homogeneous solutions), the 747 mixed strategy can use the smaller range nodes to reduce the 748 total cost. This because less powerful nodes of type $t_{2}$ are 749 employed to cover small portions of the floor-plan, like corners 750 or small regions left uncovered by the larger range nodes.

751 The amount of saving in the total cost of the mixed solu752 tion does not depend only on the nodes range and price, but 753 also on the irregularity of the floor plan perimeter. A distin754 guish feature of the proposed tool respect to other works is 755 the possibility to cover spaces that are not necessarily rectan756 gular or squared. The level of irregularity of a floor plan can 757 be identified by the minimum number of rectangles that com758 pose the shape. In Fig. 17 for example, the index of the floor 759 plan irregularity is $I=4$. We experimented the behavior of 760 the tool increasing the level of irregularity, while maintaining 761 a constant total area of $1000 \mathrm{~m}^{2}$. The test has been done with 762 the same nodes configuration used in Table V (homogeneous ${ }_{763} T=t_{1}$, mixed $\left.T=t_{1}, t_{2}\right)$. The results shown in Table VI 764 proven that increasing the floor-plan irregularity, the cost dif765 ference between homogeneous and mixed solution becomes 766 higher. This is caused by the increasing number of corners in 767 the map, that can be covered with less powerful nodes.

768 In conclusion, experimental results show that for most of 769 the problem instances, a solution can be obtained in reason770 able execution times. Depending on the available hardware 771 types, homogeneous solutions could be improved with the 772 employment of different type of nodes.

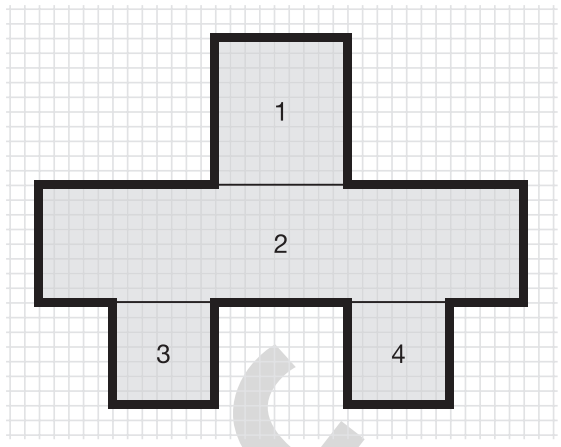

Fig. 17. Irregularity of the floor-plan perimeter summarized by the minimum number of rectangles.

TABLE VI

Cost DifFEREnCES (IN \$) BETWEen HomogeneOUS AND MiXed SOLUTION INCREASING THE FLOOR PLAN IRREGULARITY (AREA FIXED TO $1000 \mathrm{~m}^{2}$ )

\begin{tabular}{|c|cc|cc|cc|}
\hline \multirow{2}{*}{$I$} & \multicolumn{2}{|c|}{ Single } & \multicolumn{2}{c|}{ Trilater. } & \multicolumn{2}{c|}{ Fingerprint. } \\
\cline { 2 - 7 } & homog. & mixed & homog. & mixed & homog. & mixed \\
\hline 1 & 480 & 440 & 1440 & 1280 & 840 & 760 \\
2 & 480 & 440 & 1500 & 1320 & 840 & 780 \\
4 & 600 & 500 & 1560 & 1380 & 900 & 820 \\
8 & 720 & 580 & 1680 & 1480 & 1200 & 920 \\
\hline
\end{tabular}

VIII. CONCLUSION

773

In this paper, we tried to explain the challenges faced by 774 designers during the installation of smart building systems that 775 require the positioning of several hardware nodes. A com- 776 mon limitation of existing models is the lack of a convenient 777 way to specify geometric information of the indoor map. This 778 also leads to the employment of less accurate general models 779 for signal propagation, instead of site-specific models. The 780 design phase is made more difficult by the availability on 781 the market of different hardware nodes, with different power 782 transmissions and costs. $\quad 783$

For these reasons we propose an integrated tool for both 784 floor plan specification and node positioning, developed within 785 an open-source CAD environment extensible through plug-ins. 786 The tool is able to provide a near-optimal solution of node 787 allocations, possibly with mixed types, with the aim to reduce 788 the installation costs. The results suggest that, for most of 789 the problem instances, a solution can be obtained in a rea- 790 sonable execution time. Depending on the available hardware 791 types, total cost of the solution could be improved moving 792 from homogeneous to mixed type allocation. 793

A limitation of the proposed approach resides in the prop- 794 agation model used to compute near-optimal solutions for 795 localization systems. The model implemented is site-specific, 796 and take in consideration walls for LOS and NLOS prop- 797 agations. However, the approach do not consider refraction 798 or diffraction effects. Another limitation is the inability of 799 the system to model the signal propagation between differ- 800 ent floors of the building, managing each level independently. 801 For future work, we plan to improve the system with an 802 indoor signal propagation model able to consider refraction 803 and diffraction effects of the indoor environment like walls 804 and floors. In addition, we will try to apply the model to 805 
806 3-D designing tools, becoming suitable also for multifloor 807 environments.

\section{REFERENCES}

[1] C.-A. Roulet, "Indoor environment quality in buildings and its impact on outdoor environment," Energy Build., vol. 33, no. 3, pp. 183-191, 2001.

[2] V. L. Erickson, M. Á. Carreira-Perpiñán, and A. E. Cerpa, "OBSERVE: Occupancy-based system for efficient reduction of HVAC energy," in Proc. 10th ACM/IEEE Int. Conf. Inf. Process. Sensor Netw., Chicago, IL, USA, 2011, pp. 258-269. [Online]. Available: http://ACMBuildSys2015.com

[3] B. Balaji, J. Xu, A. Nwokafor, R. Gupta, and Y. Agarwal, "Sentinel: Occupancy based HVAC actuation using existing WiFi infrastructure within commercial buildings," in Proc. 11th ACM Conf. Embedded Netw. Sensor Syst., Rome, Italy, 2013, p. 17.

4] Y. Zhao, H. Zhou, and M. Li, "Indoor access points location optimization using differential evolution," in Proc. Int. Conf. Comput. Sci. Softw. Eng., Wuhan, China, 2008, pp. 382-385. [Online]. Available: http:// ieeexplore.ieee.org/lpdocs/epic03/wrapper.htm?arnumber $=4721767$

[5] Y. He, W. Meng, L. Ma, and Z. Deng, "Rapid deployment of APs in WLAN indoor positioning system," in Proc. 6th Int. ICST Conf. Commun. Netw. China (CHINACOM), Harbin, China, 2011, pp. 268-273.

6] S.-H. Fang and T.-N. Lin, "A novel access point placement approach for WLAN-based location systems," in Proc. IEEE Wireless Commun. Netw. Conf. (WCNC), Sydney, NSW, Australia, 2010, pp. 1-4.

7] ArchiCAD—The Architectural BIM CAD Software. [Online]. Available: http://www.graphisoft.com/archicad/

[8] J. P. Zhang and Z. Z. Hu, "BIM-and 4D-based integrated solution of analysis and management for conflicts and structural safety problems during construction: 1. Principles and methodologies," Autom. Construct., vol. 20, no. 2, pp. 167-180, 2011.

[9] Y. G. Xu, C. Qian, W.-P. Sung, J. C. M. Kao, and R. Chen, "Lean cost analysis based on BIM modeling for construction project," Front. Mech. Eng. Mater. Eng. II, vols. 457-458, pp. 1444-1447, 2014. [Online]. Available: http://www.scientific.net/AMM.457-458.1444.pdf

42 [10] M. S. Daskin, "A maximum expected covering location model: Formulation, properties and heuristic solution," Transp. Sci., vol. 17, no. 1, pp. 48-70, 1983. [Online]. Available: http://www.scopus.com/ inward/record.url?eid=2-s2.0-0020707868 $\{\&\}$ partnerID=tZOtx3y 1

846 [11] M. S. Daskin and E. H. Stern, "A hierarchical objective set covering model for emergency medical service vehicle deployment," Transp. Sci., vol. 15, no. 2, pp. 137-152, 1981. [Online]. Available: http://www.scopus.com/inward/record.url?eid=2-s2.0-0019565514\{\&\} partnerID=tZOtx3y 1

51 [12] V. T. Quang and T. Miyoshi, "An algorithm for sensing coverage problem in wireless sensor networks," in Proc. IEEE Sarnoff Symp., Princeton, NJ, USA, 2008, pp. 1-5.

54 [13] A. M.-C. So and Y. Ye, "On solving coverage problems in a wireless sensor network using Voronoi diagrams," in Lecture Notes in Computer Science (Including Subseries Lecture Notes in Artificial Intelligence and Lecture Notes in Bioinformatics) (LNCS 3828). Heidelberg, Germany: Springer, 2005, pp. 584-593.

9 [14] T. Andersson. (2014). Bluetooth Low Energy and Smartphones for Proximity-Based Automatic Door Locks. [Online]. Available: http://www.diva-portal.org/smash/record.jsf?pid=diva2:723899\{\&\} dswid=-9677

[15] A. S. Paul et al., "MobileRF: A robust device-free tracking system based on a hybrid neural network HMM classifier," in Proc. ACM Int. Joint Conf. Pervasive Ubiquitous Comput., Seattle, WA, USA, 2014, pp. 159-170. [Online]. Available: http://doi.acm.org/10.1145/2632048.2632097

[16] V. L. Erickson, S. Achleitner, and A. E. Cerpa, "POEM: Power-efficient occupancy-based energy management system," in Proc. 12th Int. Conf. Inf. Process. Sensor Netw., Philadelphia, PA, USA, 2013, pp. 203-216.

[17] A. Beltran, V. V. L. Erickson, and A. E. A. Cerpa, "ThermoSense: Occupancy thermal based sensing for HVAC control," in Proc. 5th ACM Workshop Embedded Syst. Energy Efficient Build., Rome, Italy, 2013, pp. 1-8. [Online]. Available: http://doi.acm.org/10.1145/2528282.252 8301\$ndelimiter”026E30F\$nhttp://dl.acm.org/citation.cfm?id=2528301

876 [18] Y. Zhao, A. LaMarca, and J. R. Smith, "A battery-free object localization and motion sensing platform," in Proc. ACM Int. Joint Conf. Pervasive Ubiquitous Comput. UbiComp Adjunct, Seattle, WA, USA, 2014, pp. 255-259. [Online]. Available: http://dx.doi.org/ 10.1145/2632048.2632078\$ndelimiter"026E30F\$nhttp://dl.acm.org/ citation.cfm?doid $=2632048.2632078$

[19] A. Corna, L. Fontana, A. A. Nacci, and D. Sciuto, "Occupancy detection 882 via iBeacon on android devices for smart building management," in 883 Proc. Des. Autom. Test Eur. Conf. Exhibit., Grenoble, France, 2015, 884 pp. 629-632.

[20] P. Kyösti et al., "IST-4-027756 WINNER II D1. 1.2 V1. 2 WINNER 886 II channel models.pdf," Projectscelticinitiativeorg, vol. 1, no. 82, 887 p. 82, 2008. [Online]. Available: http://projects.celtic-initiative.org/ 888 winner+/WINNER2-Deliverables/D1.1.2v1.2.pdf

[21] F. Colombo, R. Cordone, and G. Lulli, "The multimode covering loca- 890 tion problem," Comput. Oper. Res., vol. 67, pp. 25-33, Mar. 2016. 891 [Online]. Available: http://dx.doi.org/10.1016/j.cor.2015.09.003 892

[22] M. Kouakou, S. Yamamoto, K. Yasumoto, and M. Ito "Cost-efficient 893 deployment for full-coverage and connectivity in indoor 3D WSNs," in 894 Proc. IPSJ Dicomo, 2010, pp. 1975-1982.

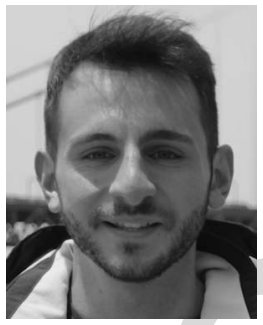

Andrea Cirigliano received the B.Sc. degree 896 in computer engineering from the Politecnico di 897 Milano, Milan, Italy, in 2013, where he is currently 898 pursuing the M.Sc. degree. 899

He joined the NECST Laboratory, Politecnico di 900 Milano, in 2015, where he is currently research- 901 ing on nonintrusive indoor localization systems and 902 occupancy detection systems. His current research 903 interests include design of smart building systems, 904 wireless indoor localization, and pervasive data 905 management.

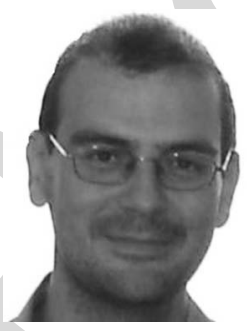

Roberto Cordone received the Dr.Eng. degree 907 in electronic engineering and the Ph.D. degree 908 in computer science and control theory from the 909 Politecnico di Milano, Milan, Italy, in 1996 and 910 2000 , respectively.

$\mathrm{He}$ is currently an Assistant Professor with the 912 Universita' degli Studi di Milano, Milan. His cur- 913 rent research interests include operations research 914 and algorithm design and analysis. $\quad 915$

Dr. Cordone is a member of the Italian 916 Association of Operations Research. $\quad 917$

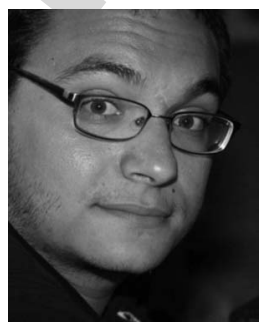

Alessandro A. Nacci received the B.Sc. and 918 M.Sc. degrees in computer engineering from the 919 Politecnico di Milano, Milan, Italy, in 2009 and 920 2012, respectively, where he is currently pursuing 921 the Ph.D. degree.

He was with EPFL, Lausanne, Switzerland. He 923 was with the Telecom Italia Joint Open Laboratory 924 S-Cube and the NECST Laboratory on the smart 925 buildings topic with the Politecnico di Milano, where 926 he has been a Research Affiliate and a Teaching 927 Assistant, since 2016. He was a Post-Doctoral 928 Research Fellow with the University of California at San Diego, San Diego, 929 CA, USA, for six months, researching at the Synergy Laboratory on the smart 930 complex buildings. In 2014, he started two companies within the Internet of 931 Things and smart building market.

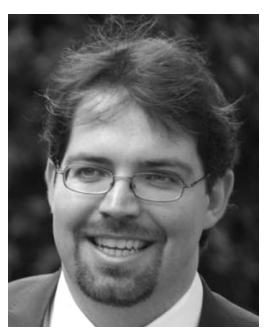

Marco Domenico Santambrogio (SM'XX) 933 AQ7 received the laurea (M.Sc. equivalent) degree in 934 computer engineering from the Politecnico di 935 Milano, Milan, Italy, in 2004, the second M.Sc. 936 degree in computer science from the University of 937 Illinois at Chicago, Chicago, IL, USA, in 2005, and 938 the $\mathrm{Ph} . \mathrm{D}$. degree in computer engineering from the 939 Politecnico di Milano, in 2008.

$\mathrm{He}$ is an Assistant Professor with the Politecnico 941 di Milano. He was a Post-Doctoral Fellow with 942 CSAIL, MIT, Cambridge, MA, USA, and has also 943 held visiting positions at the Department of Electrical Engineering and 944 Computer Science, Northwestern University, Evanston, IL, USA, in 2006945 and 2007, and Heinz Nixdorf Institute, Paderborn, Germany, in 2006. He has 946 been with the NECST Laboratory, Politecnico di Milano, where he founded 947 the Dynamic Reconfigurability in Embedded System Design project in 2004948 and the CHANGE (self-adaptive computing system) project in 2010. His 949 current research interests include reconfigurable computing, self-aware and 950 autonomic systems, hardware/software co-design, embedded systems, and 951 high performance processors and systems.

Dr. Santambrogio is a Senior Member of ACM. 


\section{AUTHOR QUERIES \\ AUTHOR PLEASE ANSWER ALL QUERIES}

PLEASE NOTE: We cannot accept new source files as corrections for your paper. If possible, please annotate the PDF proof we have sent you with your corrections and upload it via the Author Gateway. Alternatively, you may send us your corrections in list format. You may also upload revised graphics via the Author Gateway.

AQ1: Please confirm that the e-mail id for the author "A. Cirigliano" is correct as set.

AQ2: Please provide the in-text citation for "Tables I and III," "Figs. 1 and 2," and "Algorithm 1."

AQ3: Please provide the accessed date for Reference [7].

AQ4: Please provide the issue number or month for Reference [9].

AQ5: Please confirm that the edits made to References [10]-[13] and [22] are correct as set.

AQ6: Please confirm that the location and publisher information for Reference [13] is correct as set.

AQ7: Please provide the membership year for the author "M. D. Santambrogio." 